\title{
RESEARCH
}

Open Access

\section{The miR-204-5p/FOXC1/GDF7 axis regulates the osteogenic differentiation of human adipose-derived stem cells via the AKT and p38 signalling pathways}

You Zhou', Siyu Liu', Wei Wang ${ }^{2}$, Qiang Sun ${ }^{1}$, Mengzhu Lv', Shude Yang ${ }^{1}$, Shuang Tong ${ }^{1}$ and Shu Guo ${ }^{* *}$

\begin{abstract}
Background: Human adipose-derived stem cells (hADSCs) are stem cells with the potential to differentiate in multiple directions. miR-204-5p is expressed at low levels during the osteogenic differentiation of hADSCs, and its specific regulatory mechanism remains unclear. Here, we aimed to explore the function and possible molecular mechanism of miR-204-5p in the osteogenic differentiation of hADSCs.

Methods: The expression patterns of miR-204-5p, Runx2, alkaline phosphatase (ALP), osteocalcin (OCN), forkhead box C1 (FOXC1) and growth differentiation factor 7 (GDF7) in hADSCs during osteogenesis were detected by qRTPCR. Then, ALP and alizarin red staining (ARS) were used to detect osteoblast activities and mineral deposition. Western blotting was conducted to confirm the protein levels. The regulatory relationship among miR-204-5p, FOXC1 and GDF7 was verified by dual-luciferase activity and chromatin immunoprecipitation (ChIP) assays.

Results: miR-204-5p expression was downregulated in hADSC osteogenesis, and overexpression of miR-204-5p suppressed osteogenic differentiation. Furthermore, the levels of FOXC1 and GDF7 were decreased in the miR-204$5 p$ mimics group, which indicates that miR-204-5p overexpression suppresses the expression of FOXC1 and GDF7 by binding to their 3'-untranslated regions (UTRs). Overexpression of FOXC1 or GDF7 improved the inhibition of osteogenic differentiation of hADSCs induced by the miR-204-5p mimics. Moreover, FOXC1 was found to bind to the promoter of miR-204-5p and GDF7, promote the deacetylation of miR-204-5p and reduce the expression of miR-204-5p, thus promoting the expression of GDF7 during osteogenic differentiation. GDF7 induced hADSC osteogenesis differentiation by activating the AKT and P38 signalling pathways.
\end{abstract}

Conclusions: Our results demonstrated that the miR-204-5p/FOXC1/GDF7 axis regulates the osteogenic differentiation of hADSCs via the AKT and p38 signalling pathways. This study further revealed the regulatory mechanism of hADSC differentiation from the perspective of miRNA regulation.

Keywords: Human adipose-derived stem cells, Osteogenic differentiation, miR-204-5p, Forkhead box C1, Growth differentiation factor 7

\footnotetext{
* Correspondence: sguo@cmu.edu.cn

'Department of Plastic Surgery, The First Hospital of China Medical

University, NO 155 Nanjing street Heping Strict, Shenyang 110001, Liaoning

Province, China

Full list of author information is available at the end of the article
}

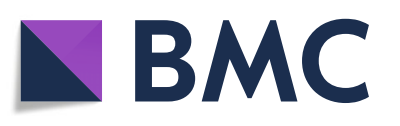

(- The Author(s). 2021 Open Access This article is licensed under a Creative Commons Attribution 4.0 International License, which permits use, sharing, adaptation, distribution and reproduction in any medium or format, as long as you give appropriate credit to the original author(s) and the source, provide a link to the Creative Commons licence, and indicate if changes were made. The images or other third party material in this article are included in the article's Creative Commons licence, unless indicated otherwise in a credit line to the material. If material is not included in the article's Creative Commons licence and your intended use is not permitted by statutory regulation or exceeds the permitted use, you will need to obtain permission directly from the copyright holder. To view a copy of this licence, visit http://creativecommons.org/licenses/by/4.0/. The Creative Commons Public Domain Dedication waiver (http://creativecommons.org/publicdomain/zero/1.0/) applies to the data made available in this article, unless otherwise stated in a credit line to the data. 


\section{Introduction}

Bone tissue engineering combines seed cells with biomaterials under the action of osteogenic inducing factors to differentiate seed cells into osteoblasts and achieve bone regeneration [1]. Therefore, seed cells are the primary link and basic elements of bone tissue engineering. hADSCs are some of the most widely used seed cells in bone tissue engineering [2]. Zuk et al. [3] first isolated hADSCs from human adipose tissue. hADSCs can be conveniently accessed by aspirating adipose tissue. Gronthos et al. have shown that resting stage hADSCs constitute $70 \%$ of the total number of cells with stem cell properties, and existing research has shown that hADSCs have equal multi-directional differentiation potential. They can differentiate into adipocytes, cardiomyocytes, nerve cells, osteoblasts, chondrocytes and so on [4]. hADSCs have many biological characteristics, such as self-renewal, multi-directional differentiation, specific migration to the injury site, and functions for tissue repair and regeneration [5]. These advantages make them favoured for clinical application, but the mechanisms of the osteogenic differentiation of hADSCs are far less understood.

MicroRNAs are a class of small molecule RNAs, approximately $22 \mathrm{nt}$ in length, that are endogenously expressed in eukaryotes [6]. They can inhibit target gene transcription or mRNA degradation by binding to the target gene [7]. MicroRNAs are involved in the regulation of a variety of biological processes, including cell proliferation and differentiation, metabolic apoptosis, DNA damage repair, cancer development, embryonic development, tissue differentiation and formation, and other physiological processes $[8,9]$. Studies have reported that microRNAs can regulate the osteogenic differentiation of mesenchymal stem cells (MSCs) [10, 11], and to the best of our knowledge, at least 27 microRNAs have been reported to participate in the osteogenic differentiation of ADSCs. miR-20a has been reported to play a positive role in regulating the osteogenic differentiation of hADSCs by co-regulating the BMP signalling pathway, while miR-23a inhibits osteogenic differentiation by acting on the key transcription factor Runx 2 $[12,13]$. miR-204-5p is a member of the miR-204 family, which is derived from the $5^{\prime}$ end arm of the hsa-miR204 precursor (pre-miRNA) [14]. Studies have shown that miR-204 is significantly downregulated during osteogenic differentiation and inhibits osteogenic differentiation by downregulating the important transcription factor Runx2 during osteogenic differentiation [15]. Nevertheless, the specific mechanism by which miR-204 regulates the osteogenic differentiation of hADSCs remains largely unknown. Moreover, complex feedback regulation exists between these miRNAs and their target osteogenic genes [11], so the regulation of the expression and degradation of these miRNAs in the differentiation process of hADSCs is worth further study.

Forkhead box $\mathrm{C} 1$ (FOXCl), a member of the fox superfamily, is widely present in various tissues and organs and regulates biological processes, including cell differentiation, embryonic development, tumorigenesis and development [16]. During embryonic development, FOXC1 activates target gene transcription by binding to the promoter of the target gene or interacting with other transcription factors [17]. FOXC1 is an important regulator of the normal development and formation of bones originating from both endochondral and intramembranous origins [18]. The Foxc1 ${ }^{-/-}$mutant mice showed a large number of abnormalities in the skulls, vertebrae, ribs and appendage bones $[19,20]$. A recent analysis showed that the ossification centres in the long bones of $\mathrm{Foxc}^{-1-}$ mutant mice were reduced, and Foxc1 regulated the PTHrP expression through interactions with Indian Hedgehog (Ihh)-Gli2 signalling, suggesting that FOXC1 played a role in regulating chondrocyte proliferation and differentiation [21]. Growth differentiation factor 7 (GDF7), also known as bone morphogenetic protein 12 (BMP12) and cartilage-derived morphogenetic protein 3 (CDMP3), is a member of the transforming growth factor beta (TGF $\beta$ ) superfamily $[22,23]$. Shen et al. found that after GDF7 stimulated ADSCs, it could promote the osteogenic differentiation of ADSCs through the Smad1/5/8 pathway [24]. However, the roles of FOXC1 and GDF7 in the osteogenic differentiation of hADSCs are still unclear, and the mechanisms also need to be further investigated.

Studies have reported that GDF7 and FOXC1 are highly expressed during hADSC osteogenic differentiation, and miR-204-5p is expressed at low levels during osteogenic differentiation [25, 26]. Moreover, TargetScan/miRDB predicts that both GDF7 and FOXC1 might be the target genes of miR-204-5p, and the JASPAR software shows that the transcription factor FOXC1 is able to bind to the promoter of miR-204-5p and GDF7. Therefore, we speculated that miR204-5p might act on the mRNA of GDF7 and FOXC1 to inhibit the osteogenic differentiation of hADSCs, and FOXC1 might bind to the promoters of miR-204-5p and GDF7 and reduce the expression of miR-204-5p, thus promoting the expression of GDF7. In this study, we aimed to investigate the role of the miR-204-5p/FOXC1/GDF7 regulatory axis in hADSC osteogenic differentiation. Our data indicated that the miR-204-5p/FOXC1/GDF7 axis formed cyclic regulation, which plays an important role in the balance of osteogenic differentiation. To our knowledge, the relationship among miR-204-5p, FOXC1 and GDF7 has not been studied during the osteogenic differentiation of hADSCs. Our data are expected to provide new insight into the differentiation of hADSCs in bone regeneration and promote hADSC clinical application. 


\section{Materials and methods}

\section{Cell culture and osteogenic induction}

hADSCs used in this study were all derived from discarded adipose tissue after liposuction from patients in the Department of Plastic Surgery, The First Hospital of China Medical University. The samples were from 8 female patients aged $29.56 \pm 5.85$ years without metabolic disease, syphilis, HIV, hepatitis or other systemic diseases. The surgical site was the abdomen, and the surgical method was suction. The above experimental samples were collected with the informed consent of the clinical patients and approved by the Ethics Committee of the First Hospital of China Medical University.

The adipose tissue obtained by liposuction of subcutaneous adipose tissue was added into $50-\mathrm{mL}$ centrifuge tubes and washed with sterile phosphate-buffered saline (PBS; Gibco, Grand Island, NY, USA). Then, the samples were centrifuged at $1000 \mathrm{~g}$ for $5 \mathrm{~min}$ to remove residual blood cells and tissue debris. The above procedure was repeated 3-4 times before enzymatic digestion with $0.2 \%$ collagenase type I (Sigma-Aldrich, St. Louis, MO, USA) at $37^{\circ} \mathrm{C}$ for $45 \mathrm{~min}$. Dulbecco's modified Eagle's medium/ Nutrient F-12 Ham (DMEM/F12 (HyClone, USA)), containing $10 \%$ foetal bovine serum (FBS (Gibco, USA)), was added to the digested lipoaspirates for $5 \mathrm{~min}$ to neutralize enzyme activity, followed by centrifugation at $1000 \mathrm{~g}$ for 5 min. Finally, the cells were plated in $75-\mathrm{cm}^{2}$ culture flasks and incubated in a culture medium (DMEM/F12, 10\% FBS, $1 \%$ penicillin-streptomycin solution (Gibco, USA)) at $37^{\circ} \mathrm{C}$ in $5 \% \mathrm{CO}_{2}$ with saturated humidity. The medium was changed for the first time after $24 \mathrm{~h}$ and then every 2 days after. hADSCs were passaged until they were $90 \%$ confluent; $0.25 \%$ trypsin:0.2\% EDTA at a ratio of $1: 3$ was used to dissociate the cells. hADSCs at passage 3 were used for subsequent experiments. When the cells adhered to the wall and grew to approximately 90\% confluence, they were cultured in groups with proliferation medium (PM) or complete OriCell osteogenic differentiation medium (Cyagen, USA) (OM).

\section{Flow cytometric analysis}

When hADSCs grew to $80 \%$ confluence, the old culture medium was removed, and the cells were washed once or twice with PBS. Trypsin solution (Gibco, USA) was added, and the cells were placed in an incubator at $37^{\circ} \mathrm{C}$ for $1 \mathrm{~min}$ and observed under an inverted microscope. The cells separated and presented granular circles with the addition of the complete medium of fresh stem cells and were blown evenly with a pipette to prepare a single-cell suspension. The single-cell suspension was transferred to a flow tube, washed with PBS twice and centrifuged at $1000 \mathrm{rpm}$ for $5 \mathrm{~min}$. The supernatant was discarded, and the cells were resuspended with $500 \mu \mathrm{L}$ $1 \times$ binding buffer and incubated with CD44, CD45,
CD90, CD105 and HLA-DR antibody (all at 1:50 dilution) working solution (Abcam, Cambridge, MA, USA) in each group, and the homotype control group was set at the same time. After incubation, the cells were centrifuged at $1000 \mathrm{~g}$ for $5 \mathrm{~min}$. The supernatant was discarded, and the cells were resuspended in $500 \mu \mathrm{L} 1 \times$ buffer and analysed by flow cytometry with a FACSCalibur flow cytometer (Becton Dickinson, Mountain View, CA, USA). The data were analysed using the CELL Quest software.

\section{RNA oligoribonucleotides and plasmid and transfection}

The RNA oligoribonucleotides used in this study, including miR-204-5p mimics, miR-204-5p inhibitor, the small interfering RNAs (siRNAs) targeting FOXC1 (siFOXC1) or GDF7 (si-GDF7), and the miR negative control (miR-NC) and siRNA control (si-NC), were purchased from GenePharma Co. (Shanghai, China). The FOCX1 and GDF7 plasmids and the empty vector were provided by Vipotion (Guangzhou, China).

At the time of passage, the surface of the culture vessel was covered with gelatine: an appropriate amount of $0.1 \%$ gelatine was added to the culture vessel to cover the whole bottom of the culture plate. The culture vessel was placed on a super clean table, and the gelatine was discarded after $30 \mathrm{~min}$. After the culture vessel was dried, it could be used for cell inoculation. hADSCs were inoculated onto gelatine-coated plates and cultured in a culture medium containing 10\% FBS. For transfection, when hADSCs reached 70-90\% confluence, they were transfected with mimics $(100 \mathrm{nM})$ or inhibitor $(100 \mathrm{nM})$ or with the FOXC1 or GDF7 plasmids or empty vector (20 ng) or siRNA $(20 \mu \mathrm{M})$ using Lipofectamine 3000 (Invitrogen, Carlsbad, CA, USA) according to the manufacturer's procedure. Cells were cultured in PM or OM for 14 days. The old medium was discarded every 3 days, the transfection processes were repeated according to the above steps and the medium was replaced with fresh complete medium. The cells were harvested at 14 days for RNA and protein analysis.

\section{RNA extraction and quantitative polymerase chain reaction (qPCR)}

Total cellular RNA was isolated at 14 days or each time point after osteoinduction or normal culturation using TRIzol Reagent (Invitrogen) according to the manufacturer's instructions. Briefly, the extracted RNA was reverse-transcribed in the presence of a poly-A polymerase with an oligo-dT adaptor. Gene expression levels were measured by the realtime PCR detection system (Bio-Rad, Hercules, CA, USA) by SYBR Green (Bio-Rad, Hercules, CA, USA) detection. The expression of miR-204-5p, Runx2, ALP, OCN, FOXC1 and GDF7 was quantified by qPCR using SYBR Green assays (TaKaRa). GAPDH and U6 were used as internal controls 
for mRNAs and miR-204-5p. The data were calculated using the $2^{-\Delta \Delta C t}$, where RQ is the relative quantity, expressed as the fold change relative to the gene expression levels in the control samples. The primer sequences used in $\mathrm{qPCR}$ were as follows: miR-204-5p-forward 5'-ACACTCCAGCTGGG TTCCCTTTGTCATCCTAT-3', miR-204-5p-reverse 5'CTCAACTGGTGTCGTGGAGTCGGCAATTCAGTTGA GAGGCATAG-3'; Runx2-forward 5'-TGGTTACTGTCAT GGCGGGTA-3', Runx2-reverse 5'-TCTCAGATCGTTGA ACCTTGCTA-3' (101 bp, GenBank accession no. NM 001015051); ALP-forward 5' -ACTGGTACTCAGACAACG AGAT-3', ALP-reverse 5'-ACGTCAATGTCCCTGATGTT ATG-3' (97 bp, GenBank accession no. NM_003064); OCNforward 5'-CACTCCTCGCCCTATTGGC-3', OCN-reverse 5'-CCCTCCTGCTTGGACACAAAG-3' (112 bp, GenBank accession no. NM_199173); FOXC1-forward 5'-TGTT CGAGTCACAGAGGATCG-3', FOXC1-reverse 5'-ACAG TCGTAGACGAAAGCTCC-3' (122 bp, GenBank accession no. NM_001453); GDF7-forward 5'-TGATGTCGCTTTAC CGGAGC-3, GDF7-reverse 5'-CTGCCGATTCGTCTTGG GT-3' (129 bp, GenBank accession no. AB158468); GAPDHforward 5'-TGTTCGTCATGGGTGTGAAC-3', GAPDHreverse 5'-ATGGCATGGACTGTGGTCAT-3' (154 bp, GenBank accession no. AF261085); and U6-forward 5'CTCGCTTCGGCAGCACA-3', U6-reverse 5'-AACGCT TCACGAATTTGCGT-3' (96 bp, GenBank accession no. NR_004394).

\section{Western blot analysis}

After 14 days of osteoinduction or normal culture, the proteins of hADSCs were extracted by using RIPA buffer containing 1\% PMSF (Sigma-Aldrich). The BCATM Protein Assay Kit (Pierce, Appleton, WI, USA) was used for quantification of protein samples. Equal amounts of protein samples $(30 \mu \mathrm{g})$ were separated on $12 \%$ SDS-PAGE gels and transferred to PVDF membranes (Millipore Corporation, Billerica, MA, USA). After blocking in 0.5\% bovine serum albumin (BSA; Roche) for $1 \mathrm{~h}$ at $37^{\circ} \mathrm{C}$, the blocked membranes were incubated with the corresponding primary antibodies overnight at $4{ }^{\circ} \mathrm{C}$, including FOXC1 (ab223850, 1:1000), ALP (ab16695, 1:1000), RUNX2 (ab23981, $1 \mu \mathrm{g} / \mathrm{ml})$, OCN (ab93876, $1 \mu \mathrm{g} / \mathrm{ml}$ ), P38 (ab170099, 1:1000), p-P38 (ab4822, 1:500), AKT (ab179463, 1:1000), p-AKT (ab38449, 1:500) and GAPD $\mathrm{H}$ (ab181602, 1:1000), which were purchased from Abcam (Cambridge, MA, USA). The membranes were then washed and incubated with appropriate HRP secondary antibodies (ab6721, 1:2000) (Cambridge, MA, USA) for $1 \mathrm{~h}$ at $37^{\circ} \mathrm{C}$. The signals were detected and analysed by an enhanced chemiluminescence system (Amersham Biosciences, Piscataway, NJ, USA) and Image Lab software (Bio-Rad, Cal, USA). Protein levels were quantified using Image Lab software.

\section{Alkaline phosphatase (ALP) staining}

Transfected hADSCs cultured in PM or OM for 14 days were assayed for ALP staining and activity. The medium was removed after 14 days, and the cells were washed twice with PBS and fixed with $4 \%$ paraformaldehyde (Jianglaibio, Shanghai, China) for $30 \mathrm{~min}$. The paraformaldehyde was removed, and the cells were washed with $\mathrm{ddH} 2 \mathrm{O}$ three times, and alkaline phosphatase staining solution (Beyotime, China) was added for $30 \mathrm{~min}$. The ALP staining solution was removed, and the cells were washed with $\mathrm{ddH} 2 \mathrm{O}$ three times and observed under a microscope (Leica DMIRB, Germany) and imaged.

\section{Alizarin red staining (ARS)}

Transfected hADSCs cultured in PM or OM for 14 days were assayed for ARS. The culture medium was discarded, and the cells were fixed with $4 \%$ paraformaldehyde for 15-20 min and washed with PBS 3 times. ARS solution (ScienCell, San Diego, CA, USA) was prepared in advance and added to the culture plate; the plate was placed in the incubator for $15 \mathrm{~min}$, the staining solution was removed and the sample was washed with PBS solution three times; the PBS was removed, and the samples were placed under a differential microscope to take photos (Leica DMIRB, Germany).

\section{Immunofluorescence staining}

hADSCs transiently transfected with miR-204-5p mimics or inhibitors were seeded in 6-well plates. After 14 days in $\mathrm{PM}$ or $\mathrm{OM}$, cells grown on sterile glass coverslips were fixed in $4 \%$ paraformaldehyde (Jianglaibio, Shanghai, China) for $30 \mathrm{~min}$, permeabilized with $0.1 \%$ Triton $\mathrm{X}-100$ for $15 \mathrm{~min}$ and blocked with $5 \%$ normal goat serum for $30 \mathrm{~min}$. Then, cells were incubated with a primary antibody-OCN (ab13418, 1:500; Abcam) and incubated with an anti-mouse secondary antibody (ab150117, 1:500; Abcam) for $1 \mathrm{~h}$ at room temperature. 4,6-Diamidino-2-phenylindole (DAPI) was used to stain the nuclei, and the coverslips were mounted on a glass slide and observed under a confocal Zeiss Axiovert 650 microscope at $488 \mathrm{~nm}$ (green, OCN) and $405 \mathrm{~nm}$ (blue, DAPI). Images were captured using an LSM 5 Exciter confocal imaging system (Carl Zeiss).

\section{Dual-luciferase reporter assay}

Luciferase reporter assays were carried out as follows. First, the 3'-UTR sequences of wild-type (WT) and mutant-type (MUT) FOXC1 and GDF7 were cloned into the psiCHECk2 vector, while the WT and MUT regions of the promoters of miR-204-5p and GDF7 were cloned into the pGL3 vector. To analyse the interaction between miR-204-5p and FOXC1 or GDF7, hADSCs $(1 \times$ $10^{5}$ ) were grown in a 96-well plate and co-transfected with either miR-204-5p mimics (100 nM) or NC mimics 

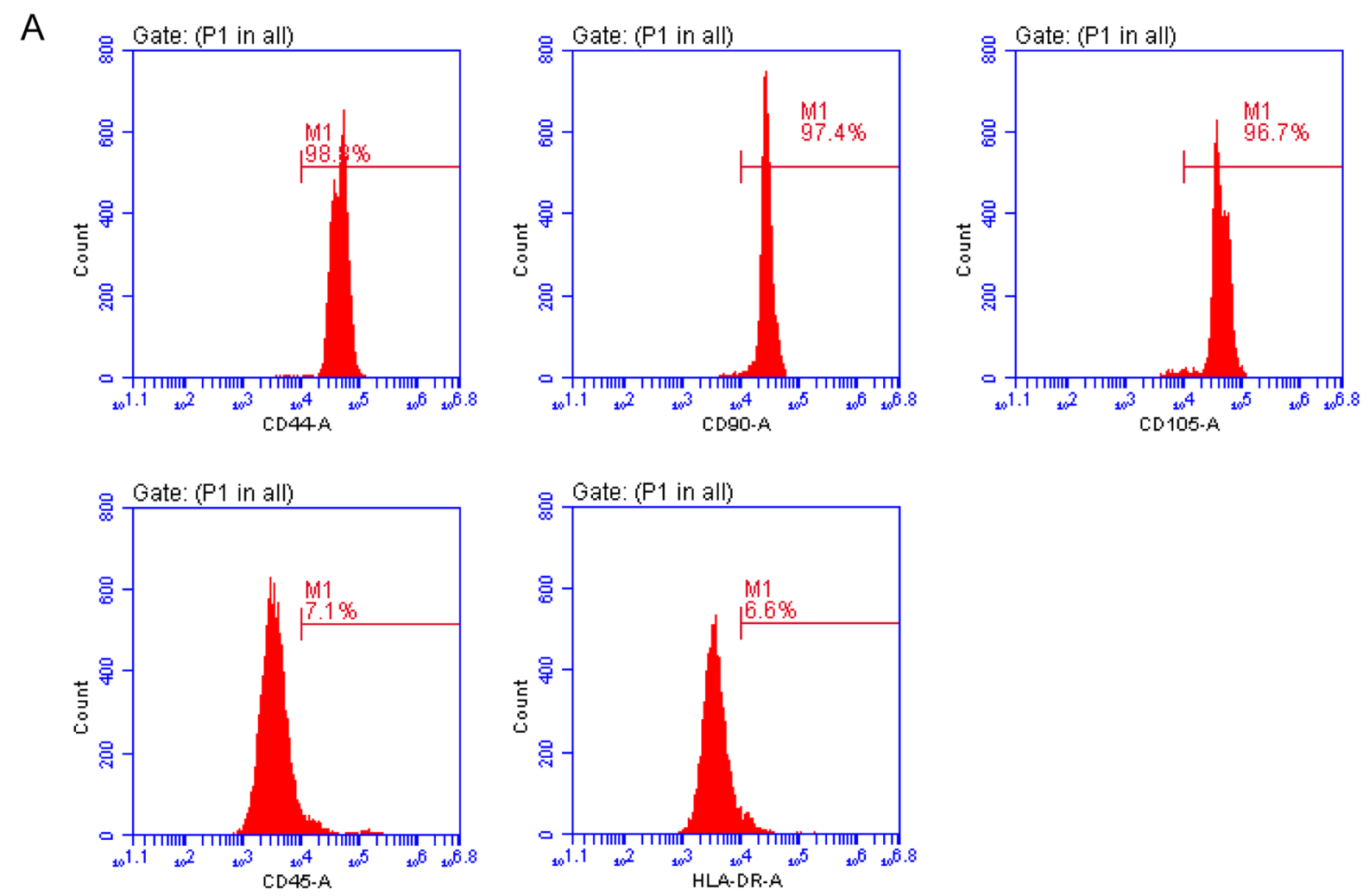

B

miR-204-5p
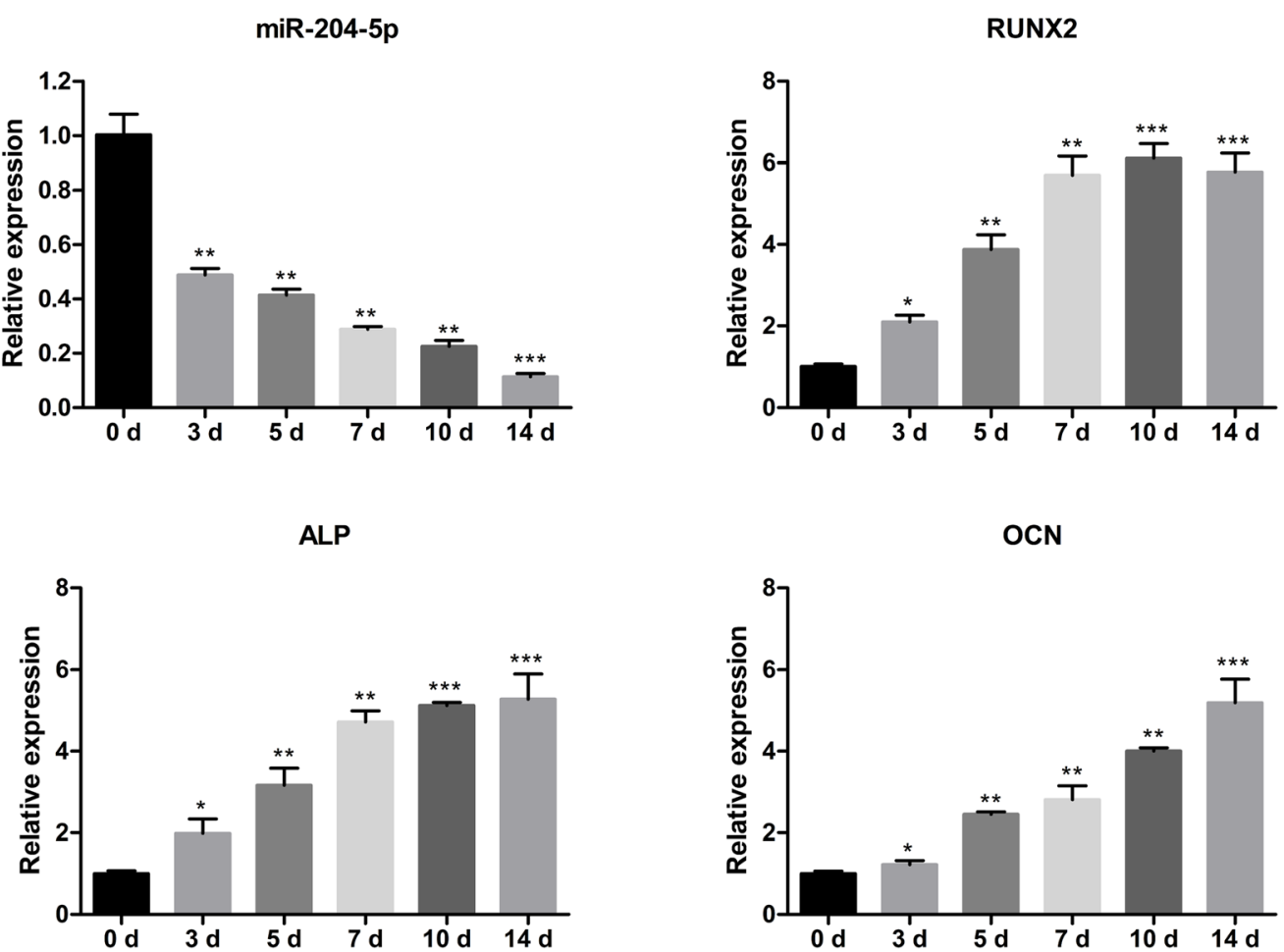

Fig. 1 (See legend on next page.) 
(See figure on previous page.)

Fig. 1 miR-204-5p is downregulated during the osteogenic differentiation of hADSCs. a Flow cytometry was used to identify hADSCs by detecting specific marker proteins CD44, CD45, CD90, CD105 and HLA-DR. b Relative expression of miR-204-5p at each time point during the osteogenic differentiation of hADSCs detected by qRT-PCR. U6 was used as a reference gene. Relative mRNA levels of the osteogenic markers RUNX2, ALP and OCN at each time point during the osteogenic differentiation of hADSCs detected by qRT-PCR. GAPDH was used as an internal control gene. Data are presented as the mean \pm SD. ${ }^{*} p<0.05,{ }^{* *} p<0.01,{ }^{* * *} p<0.01$ ( $n=3$ independent experiments)

(100 nM), FOXC1-WT/MUT (20 ng), or GDF7-WT/ MUT (20 ng) by Lipofectamine 2000 (Invitrogen). To assess FOXC1 binding to the promoter of GDF7 and miR204-5p, hADSCs were grown in a 96-well plate and cotransfected with either vector/vector-FOXC1 or miR204-5p-WT/MUT or GDF7-WT2/MUT2. Renilla and firefly luciferase activities were measured $48 \mathrm{~h}$ after transfection using the Dual-Luciferase Reporter Assay System (Promega, Beijing, China). All luciferase values were normalized to those of firefly luciferase and expressed as fold induction relative to the basal activity.

\section{Chromatin immunoprecipitation (ChIP) assay}

ChIP assays were conducted by using an EZ-Magna ChIP assay kit (Merck Millipore). hADSCs were seeded in 10$\mathrm{cm}$ dishes and transfected with FOXC1 or the vector. The cells were crosslinked with $1 \%$ formaldehyde and sonicated to shear DNA. Then, the DNA-protein complexes were isolated with antibodies against isotype immunoglobulin G (IgG) and HDAC2, H3K9AC, and FOXC1 (Cell Signaling). The protein-DNA complexes were then purified and reverse-crosslinked. The DNA was isolated and quantified by qRT-PCR. Relative enrichment was calculated as the amount of amplified DNA relative to values obtained from Input. The primer sequences used in ChIP assays were as follows: FOXC1-miR-204-5p-forward 5' TGGGGTAGTTGCCAGTTAGA-3', FOXC1-miR-204-

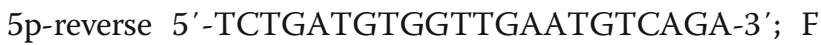
OXC1-GDF7-forward 5'-AAACACCCAAACACTGCG G-3', FOXC1-GDF7-reverse 5'-GGGATAGTCCACCC TGCTTCT-3'; H3K9ac-miR-204-5p-forward 5'-ACCA CAGAAGTCTTCATTTCCT-3', H3K9ac-miR-204-5preverse 5'-AATAGTGCCGTCAAGCTGTC-3'; and HD AC2-miR-204-5p-forward 5'-GAAGGGCTGGATGATG CTCT-3', HDAC2-miR-204-5p-reverse 5'-GCAGATGG ATTACCCAATTTACAT-3'.

\section{Statistical analysis}

All the corresponding experiments were independently repeated more than three times, and the Western blot results are representative. All data in this experiment are expressed as the mean \pm standard deviation. SPSS 17.0 for Windows statistical software was used for the Kruskal-Wallis one-way analysis of variance (ANOVA) test, and $P<0.05$ indicated a significant difference.

\section{Results}

miR-204-5p is downregulated during the osteogenic differentiation of hADSCs

As a mesenchymal lineage cell population, ADSCs express CD44, CD90 and CD105, while haematopoietic markers such as CD45 and histocompatibility leukocyte antigen (HLA)-DR are absent. hADSCs were collected and phenotypically identified, and the results showed that the cells were positive for CD44, CD90 and CD105 and negative for CD45 and HLA-DR, which suggested that the primary cultured cells were hADSCs (Fig. 1a). These identified hADSCs were cultured under osteogenic induction conditions for 14 days. Since it has been reported that miR-204 is downregulated during osteogenic differentiation, we detected the expression level of hsa-miR-204-5p by qRT-PCR at 3, 5, 7, 10 and 14 days in these identified hADSCs. Consistent with a previous report, the results showed that the expression level of miR-204-5p decreased gradually with induction time (Fig. 1b). At the same time, we detected the expression levels of the osteogenic markers RUNX2, ALP and OCN in cells at various time points. The qRT-PCR results showed that the expression levels of RUNX2, ALP and $O C N$ were markedly upregulated during osteogenesis (Fig. 1b).

\section{miR-204-5p inhibits the osteogenic differentiation of hADSCs in vitro}

To explore the effect of miR-204-5p on the osteogenic differentiation of hADSCs, we transfected miR-204-5p mimics or inhibitor into hADSCs and cultured them in PM or OM. The expression level of miR-204-5p was detected by qRT-PCR at 14 days in PM and OM, and it was increased when cells were transfected with miR204-5p mimics but decreased when cells were transfected with miR-204-5p inhibitor (Fig. 2a). qRT-PCR and Western blotting were used to detect the mRNA and protein expression levels of the osteogenic markers RUNX2, ALP and OCN. The results showed that miR204-5p mimics could decrease RUNX2, ALP and OCN expression, whereas miR-204-5p inhibitor could increase RUNX2, ALP and OCN expression (Fig. 2a, b). In addition, immunofluorescence staining indicated that the protein level of OCN was reduced in the miR-204$5 p$ overexpression group and increased in the miR-204$5 p$ inhibition group (Fig. 2c). 


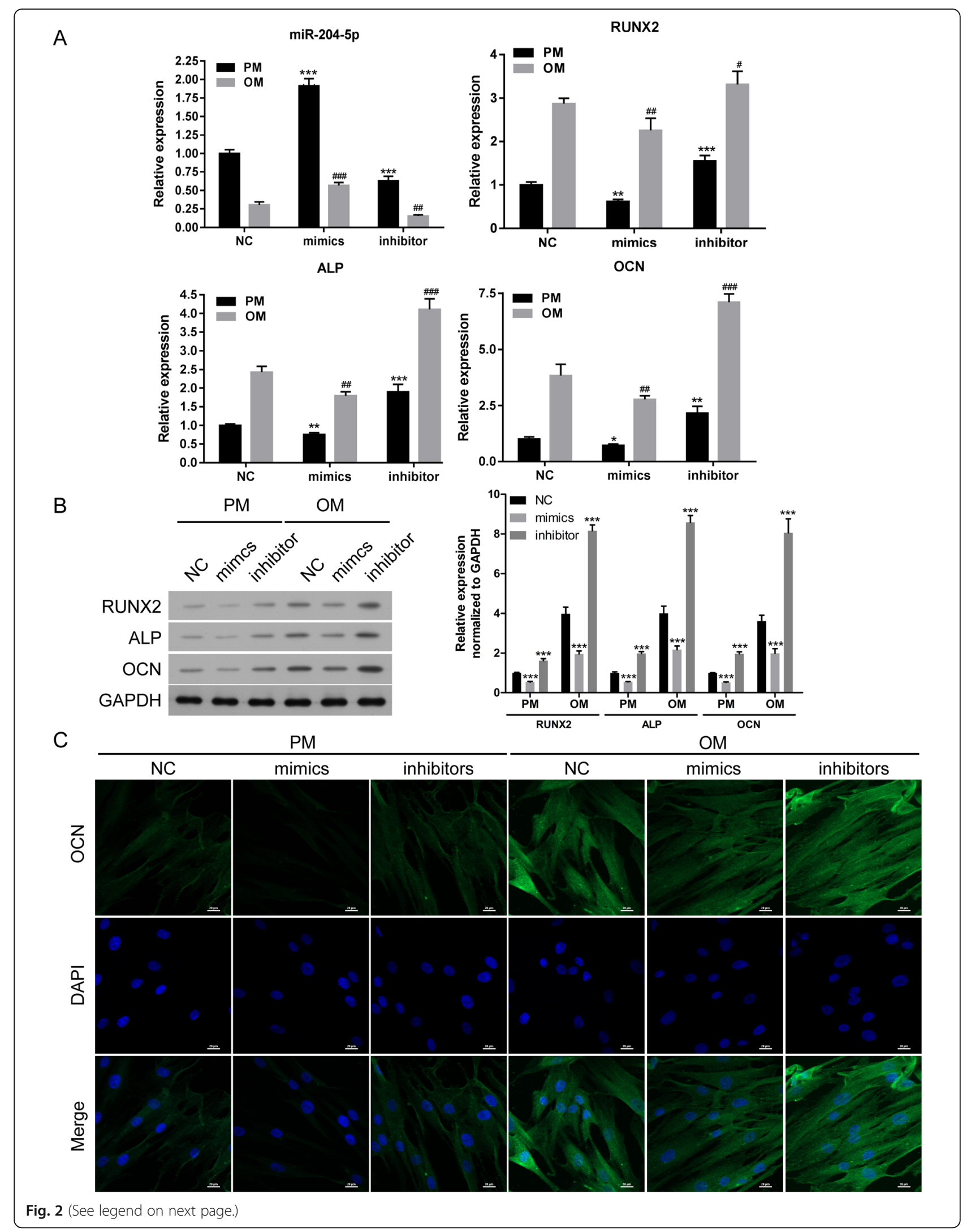


(See figure on previous page.)

Fig. 2 miR-204-5p overexpression inhibits the expression of RUNX2, ALP and OCN. hADSCs were transfected with miR-204-5p mimics, miR-204-5p inhibitors or NC and cultured in PM or OM. a Relative mRNA levels of miR-204-5p, RUNX2, ALP and OCN measured by qRT-PCR on day 14 of osteogenic induction. U6 and GAPDH were used as internal control genes. The data were normalized to the NC-PM group. $\mathbf{b}$ Left panel: Western blot of RUNX2, ALP and OCN protein levels on day 14 after osteogenic induction. GAPDH was used as an internal control. Right panel: quantitative analysis of the protein levels. c Confocal microscopy of cells with OCN with DAPI counterstaining on day 14 after osteogenic induction. Scale bars, $20 \mu \mathrm{m}$. Data are presented as the mean \pm SD. *hADSCs transfected with miR-204-5p mimics or inhibitors vs NC in PM; ; ${ }^{*}$ ADSCs transfected with miR-204-5p mimics or inhibitors vs NC in OM. ${ }^{*}$ or ${ }^{\#} p<0.05,{ }^{* *}$ or ${ }^{\# \#} p<0.01,{ }^{* * *}$ or ${ }^{\# \# \#} p<0.001$ ( $n=3$ independent experiments)

Moreover, Western blot analysis showed that the protein levels of FOXC1 and GDF7 were also decreased in the miR-204-5p mimic group and increased in the miR204-5p inhibition group (Fig. 3a).

ALP staining showed that miR-204-5p mimics suppressed the osteogenic differentiation of hADSCs, and miR-204-5p inhibitor enhanced the osteogenic differentiation of hADSCs in PM or OM (Fig. 3b). The extracellular mineralization of hADSCs was measured by ARS assay in PM or OM on day 14, and the results showed that the osteogenic differentiation of hADSCs was suppressed with miR-204-5p mimics but increased with miR-204-5p inhibitor (Fig. 3c). Taken together, these results suggested that miR-204-5p inhibits the osteogenic differentiation of hADSCs in vitro.

\section{FOXC1 reduces the suppressive effect of miR-204-5p on the osteogenic differentiation of hADSCs}

To investigate the role of FOXC1 in osteogenic differentiation, the cells were co-transfected with miR-204-5p mimics and FOXC1 plasmid or co-transfected with miR204-5p inhibitor and FOXC1 siRNA. Compared with the empty vector transfection, FOXC1 overexpression inhibited miR-204-5p expression but promoted the expression of FOXC1 and GDF7 and increased RUNX2, ALP and $O C N$ expression under miR-204-5p mimic transfection, whereas FOXC1 downregulation obtained the opposite results in hADSCs transfected with miR-204-5p inhibitor (Fig. 4a). Then, we also carried out Western blotting to evaluate the protein expression levels of FOXC1, GDF7, RUNX2, ALP and OCN and obtained the same results as with qRT-PCR (Fig. 4b). Subsequently, ALP staining and ARS showed that overexpression of FOXC1 attenuated the suppression effect of miR-204-5p on the osteogenic differentiation and extracellular mineralization of hADSCs, while downregulation of FOXC1 inhibited the osteogenic differentiation and extracellular mineralization of hADSCs induced by the miR-204-5p inhibitor (Fig. 5a, b). These results suggested that FOXC1 reduces the suppressive effect of miR-204-5p on the osteogenic differentiation of hADSCs.

To explore the relationship between miR-204-5p and FOXC1, we used TargetScan/miRDB to search for potential targets of miR-204-5p. We found that the 3 '-UTR of FOXC1 has two miR-204-5p binding sites (Fig. 5c). Next, we constructed a WT reporter and MUT reporter that contained a mutant 3 '-UTR of FOXC1 designed with mutated sequences of the miR-204-5p binding site. The results indicated that overexpression of miR-204-5p markedly decreased the luciferase activity in the WT group of FOXC1 cells, whereas this phenomenon was not observed in the MUT group (Fig. 5d), which suggested that FOXC1 is a target of miR-204-5p.

GDF7 impairs the suppressive effect of miR-204-5p on the osteogenic differentiation of hADSCs

To explore the role of GDF7 in osteogenic differentiation, hADSCs were co-transfected with miR-204-5p mimics and GDF7 plasmid or co-transfected with miR204-5p inhibitor and GDF7 siRNA. Compared with the empty vector transfection, the results showed that overexpression of GDF7 could inhibit miR-204-5p expression but promote the mRNA and protein expression of GDF7, RUNX2, ALP and OCN, whereas downregulation of GDF7 obtained opposite results (Fig. 6a, b). Recent studies proved that the AKT and P38 signalling pathways were involved in osteogenic differentiation, so we next carried out Western blotting to evaluate the protein expression levels of p-AKT, AKT, p-P38 and P38. The results suggested that overexpression of GDF7 could enhance the phosphorylation of AKT and P38 under treatment with miR-204-5p mimics, while knockdown of GDF7 reduced the phosphorylation of AKT and P38 under inhibition of miR-204-5p (Fig. 6c).

Moreover, ALP staining showed that overexpression of GDF7 promoted the osteogenic differentiation of hADSCs transfected with miR-204-5p mimics, while downregulation of GDF7 suppressed this process in hADSCs transfected with miR-204-5p inhibitor (Fig. 7a). ARS showed that overexpression of GDF7 increased the extracellular mineralization of hADSCs transfected with miR-204-5p mimics, but downregulation of GDF7 inhibited the extracellular mineralization of hADSCs transfected with miR204-5p inhibitor (Fig. 7b). Taken together, these results indicated that GDF7 impairs the suppressive effect of miR204-5p on the osteogenic differentiation of hADSCs via the AKT and p38 signalling pathways.

Finally, we used a dual-luciferase reporter assay to verify whether GDF7 is a potential target of miR-204-5p. We found that there is a miR-204-5p binding site in the 3 '-UTR of 

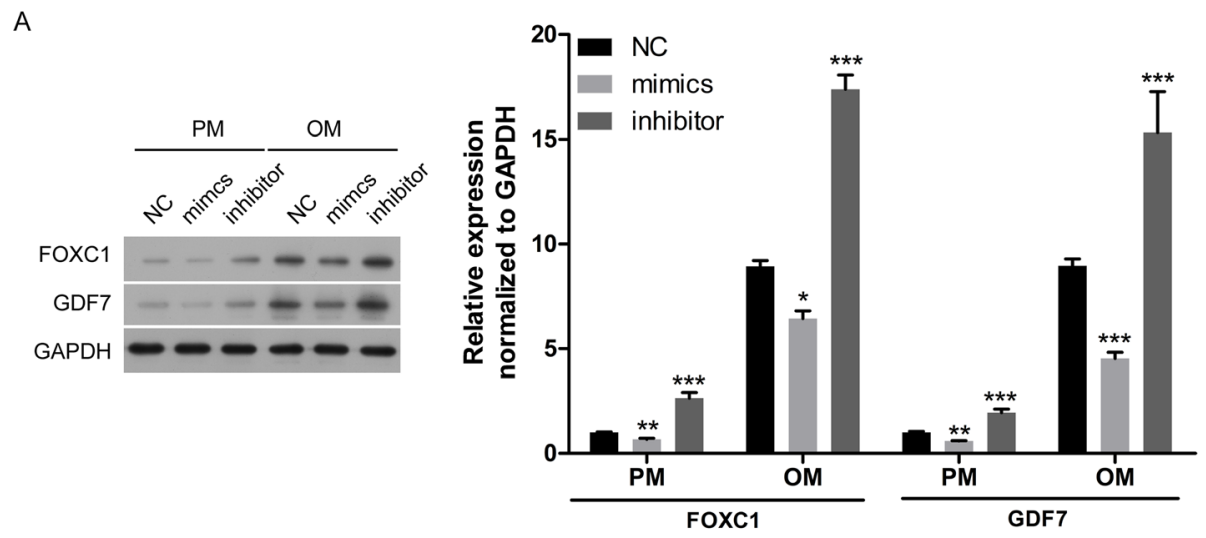

B
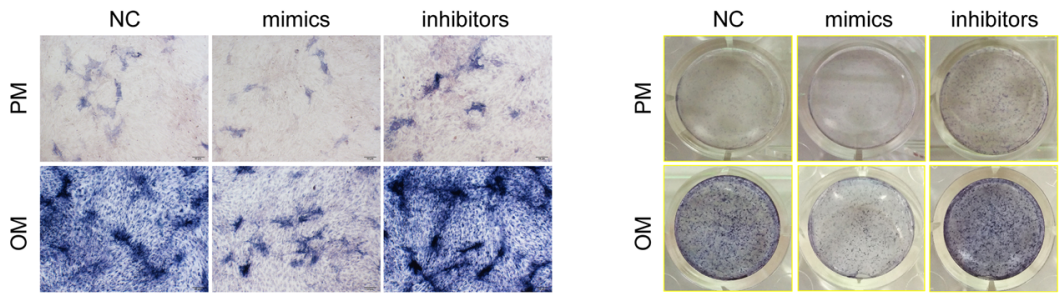

C
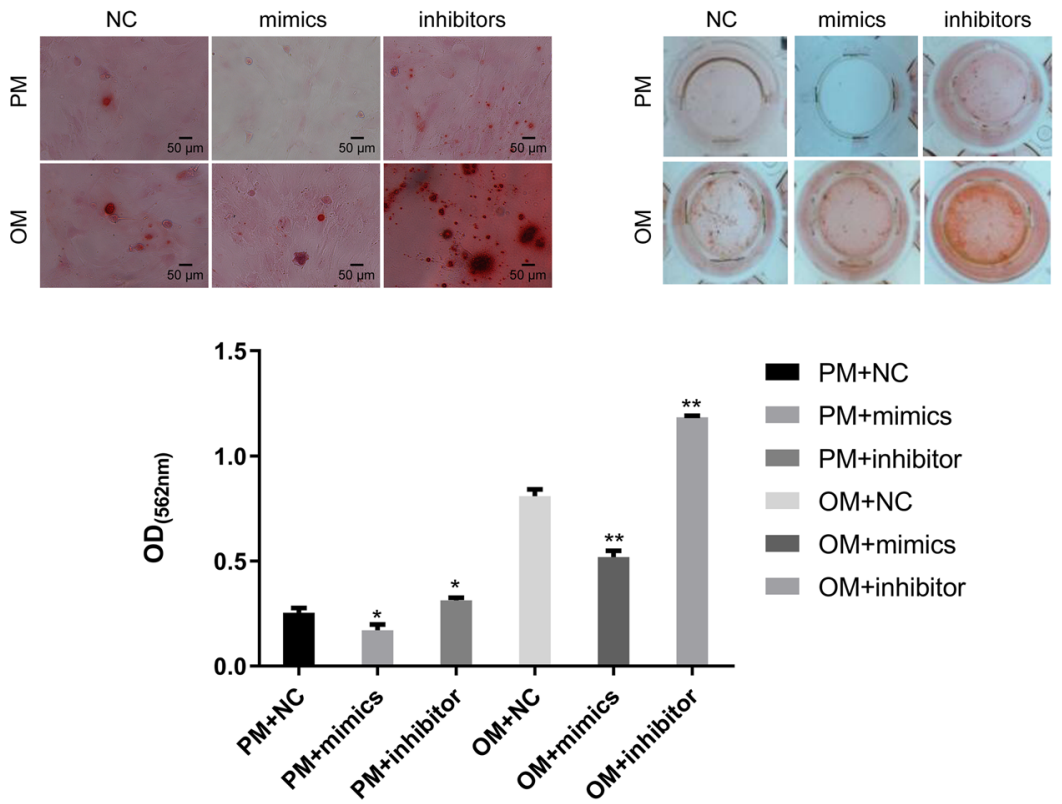

Fig. 3 miR-204-5p overexpression inhibits the osteogenic differentiation of hADSCs. hADSCs were transfected with miR-204-5p mimics, miR-204-5p inhibitors or NC and cultured in PM or OM. a Left panel: Western blot of FOXC1 and GDF7 protein levels on day 14 after osteogenic induction. GAPDH was used as an internal control. Right panel: quantitative analysis of the protein levels. ALP staining (b) and ARS (c) on day 14 after osteogenic induction. Quantification of ARS was shown below. Data are presented as the mean \pm SD. ${ }^{*} p<0.05,{ }^{* *} p<0.01,{ }^{* * *} p<0.001$ ( $n=3$ independent experiments)

GDF7 (Fig. 7c). Next, we constructed a WT reporter and MUT reporter that contained a mutant 3'-UTR of GDF7 designed with mutated sequences of the miR-204-5p binding site. The results indicated that overexpression of miR-204-5p markedly decreased the luciferase activity in the WT group of GDF7 cells but not in the MUT group (Fig. 7d). This result suggested that GDF7 is also a target of miR-204-5p.
FOXC1, as a transcription factor, could bind to the promoter of miR-204-5p and GDF7

Then, we sought to explore the specific regulatory mechanism among miR-204-5p, FOXC1 and GDF7 in the osteoblast differentiation of hADSCs. We speculated that the transcription factor FOXC1 might bind to the promoters of miR-204-5p and GDF7. As expected, a 


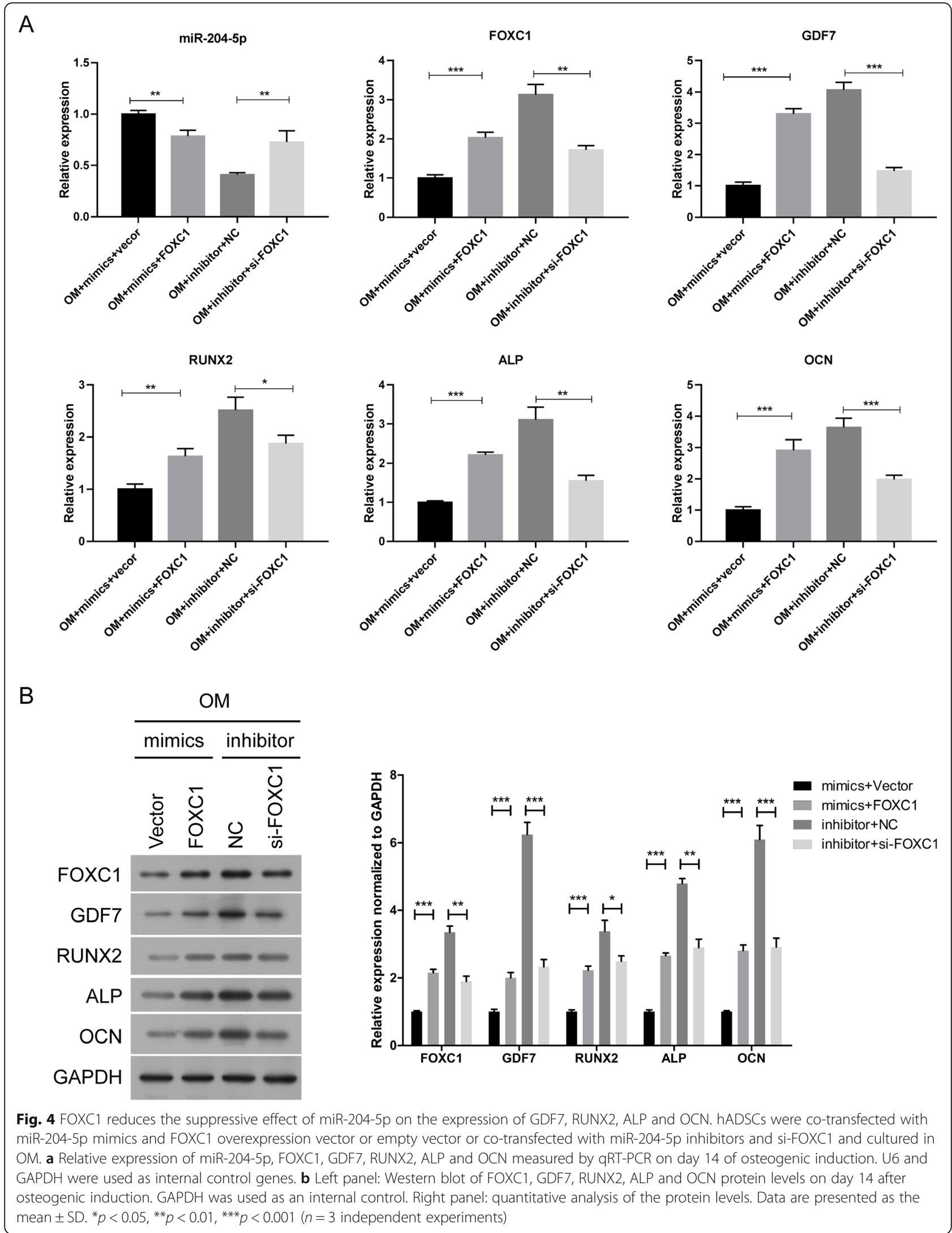


A

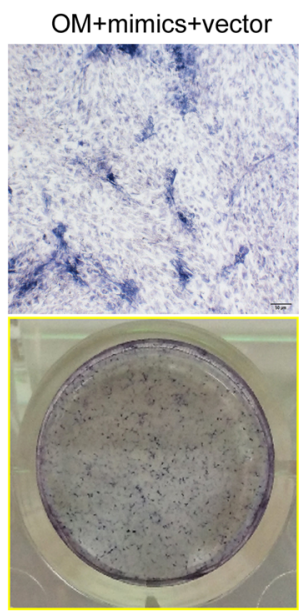

B
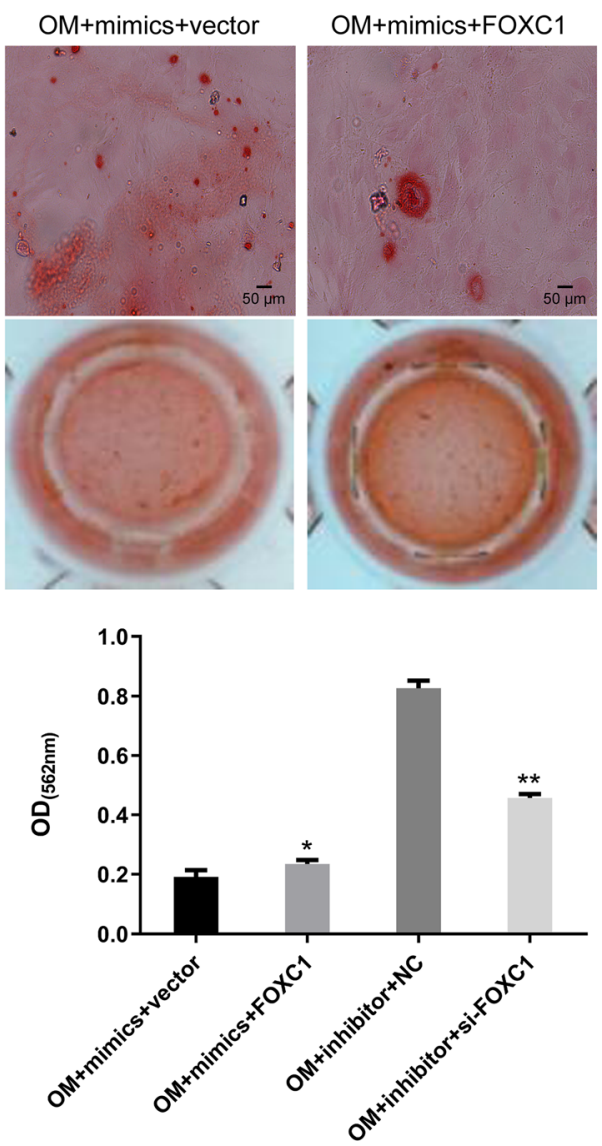

C

hsa-miR-204-5p: 3'-UCCGUAUCCUACUGUUUCCCUU-5' IIIII 3'UTR of FOXC1 (619-626): 5'-CTTTTTTTTCTCATTATAAAAAGGGA-3' hsa-miR-204-5p: 3'-UCCGUAUCCUACUGUUUCCCUU-5' 3'UTR of FOXC1(1324-1331): 5'-ATATACTGCGGGTTGGAAAGGGA-3
$\mathrm{OM}+$ inhibitor+NC
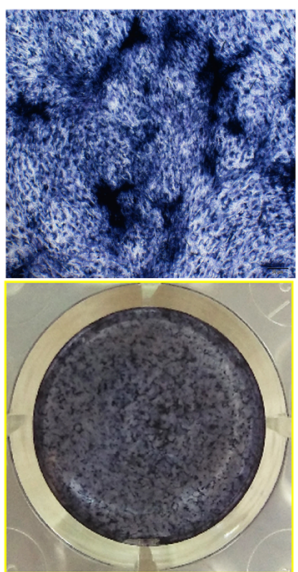

$\mathrm{OM}+$ inhibitor+NC
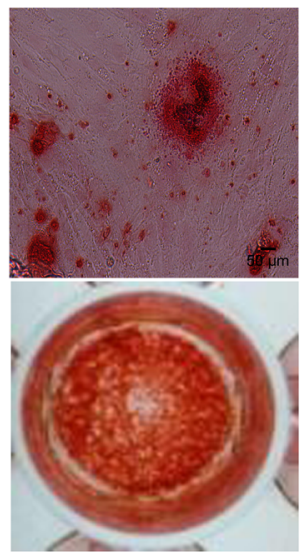

OM+inhibitor+si-FOXC1
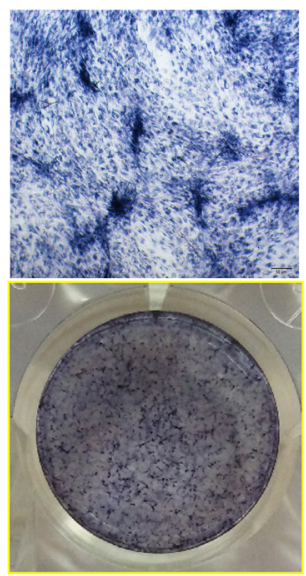

OM+inhibitor+si-FOXC1
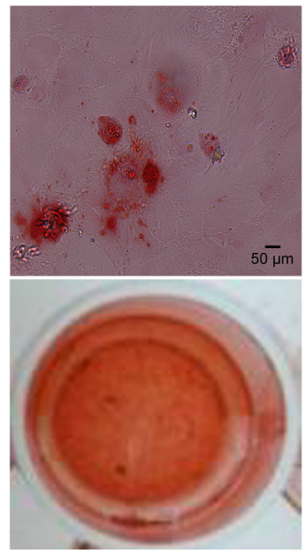

- OM+mimics+vector

OM+mimics+FOXC1

- OM+inhibitor+NC

- OM+inhibitor+si-FOXC1

$\mathrm{D}$

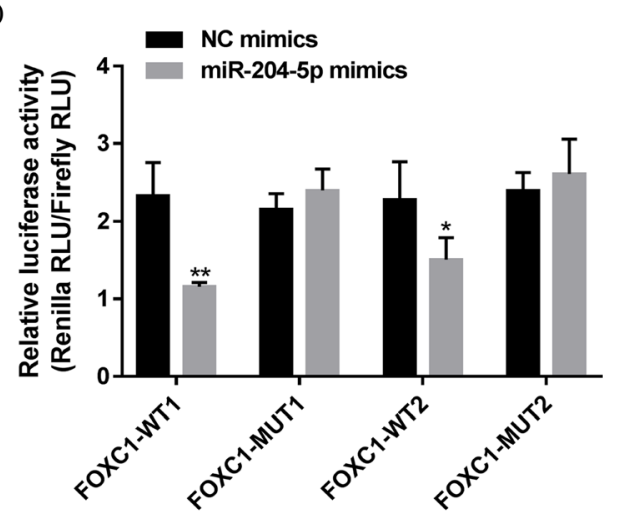

Fig. 5 (See legend on next page.) 
(See figure on previous page.)

Fig. 5 FOXC1 reduces the suppressive effect of miR-204-5p on the osteogenic differentiation of hADSCs. hADSCs were co-transfected with miR204-5p mimics and FOXC1 overexpression vector or empty vector or co-transfected with miR-204-5p inhibitors and si-FOXC1 and cultured in OM. ALP staining (a) and ARS (b) on day 14 after osteogenic induction. Quantification of ARS was shown below. c Predicted binding site of miR-204$5 p$ in the $3^{\prime}-$ UTR of FOXC1 mRNA. d Relative luciferase activity of cells in different groups. Data are presented as the mean \pm SD. ${ }^{*} p<0.05,{ }^{* *} p<$ $0.01,{ }^{* * *} p<0.001$ ( $n=3$ independent experiments)

putative binding site of FOXC1 was found in the promoters of both miR-204-5p (Fig. 8a) and GDF7 (Fig. 8c). We generated dual-luciferase reporters containing the WT or MUT promoter of miR-204-5p and GDF7. The results showed that overexpression of FOXC1 decreased the luciferase activity of the miR-204-5p promoter but increased the luciferase activity of the GDF7 promoter (Fig. 8b, d), indicating that FOXC1 regulates the promoter activity of miR-204-5p and GDF7. Referring to the predicted binding sites in Fig. 5a, c, gel electrophoresis and ChIP assays further confirmed that FOXC1 regulated the promoters of GDF7 and miR-204-5p through direct binding (Fig. 8e).

To further investigate the effect of FOXC1 on the transcriptional activity of the miR-204-5p promoter, we focused on histone acetylation regulation. Putative binding sites of histone deacetylase HDAC2 and histone acetylase H3K9AC were also found in the promoter of miR-204-5p. Gel electrophoresis and ChIP assays confirmed that both HDAC2 and H3K9AC were able to bind to the promoter of miR-204-5p. Furthermore, compared with empty vector control transfection, overexpression of FOXC1 promoted the interaction between histone deacetylase HDAC2 and miR-204-5p but inhibited the interaction between histone acetylase H3K9AC and miR-204-5p (Fig. 8f, g). These results proved that FOXC1 epigenetically silences miR-204-5p transcription through interaction with HDAC2 and H3K9AC.

\section{Discussion}

In the present study, we investigated the osteogenic differentiation of hADSCs, showing that miR-204-5p was downregulated during the osteogenic differentiation of hADSCs. Moreover, miR-204-5p can suppress the expression of FOXC1 and GDF7, and FOXC1 could reduce the expression of miR-204-5p, thus promoting the expression of GDF7 and activating the AKT and P38 signalling pathways.

Compared with other MSCs, hADSCs stand out because of their abundance in the body, less invasive extraction procedures, lower incidence rate and multidirectional differentiation potential [27]. They have become a hotspot in the field of MSCs and one of the important sources of autologous stem cell therapy [28]. In this study, we found a new chain that could accelerate the hADSC osteogenic process.
In recent years, many studies have investigated the role and mechanism of microRNAs in stem cell adipogenesis or osteogenic differentiation [10]. A previous study analysed the expression profiles of microRNAs in MSCs during osteogenic differentiation and showed that miR22 expression was upregulated during osteogenesis but reduced during MSC adipogenic differentiation [29]. Studies have reported that miR-103-3p regulates the osteogenic differentiation of bone marrow mesenchymal cells by inhibiting the target gene Satb2 [30, 31]. Moreover, a study has shown that miR-204-5p promoted the adipogenic differentiation of hADSCs by modulating DVL3 expression and suppressing $\mathrm{Wnt} / \beta$-catenin signalling [25]. In this study, we proved that overexpression of miR-204-5p could decrease RUNX2, ALP and OCN expression and suppress the osteogenic differentiation of hADSCs, whereas inhibition of miR-204-5p could increase RUNX2, ALP and OCN expression and promote the osteogenic differentiation of hADSCs, suggesting that miR-204-5p might be an important target miRNA for hADSC-based therapies related to bone regeneration.

FOXC1 is an important regulator of the initial stage of the osteogenesis process in the membrane and cartilage. Oshiki et al. proved that Foxc1 could inhibit adipogenic processes in stem cells, and subsequent research disclosed that FOXC1 could promote RUNX2 expression by binding to the RUNX2 promoter [32]. Moreover, FOXC1 is essential for the maintenance of mesenchymal niches. This effect may be mediated by BMP signalling because FOXC1 could activate the BMP pathway to govern hair follicle stem cell quiescence [33]. In addition, inactivation of FOXC1 could result in a dramatic reduction in mouse skull vault growth [18]. However, there are some opinions against the positive effect of FOXC1 in osteogenesis. Caddy et al. described that FOXC1 downregulates BMP4-induced osteoblast differentiation [34]. FOXC1 expression is regulated by microRNAs, such as miR-138-5p, miR-374c and miR-133 [35, 36]. Our data indicated that FOXC1 is the target gene of miR-204-5p and that miR-204-5p could suppress the expression of FOXC1. Similar to mainstream views, our data also showed that FOXC1 promotes hADSC osteogenic differentiation.

Moreover, we found that GDF7, also named BMP12, is the downstream key protein in FOXC1-mediated osteogenic differentiation of hADSCs. We found that overexpression of FOXC1 could promote GDF7 expression and 
A

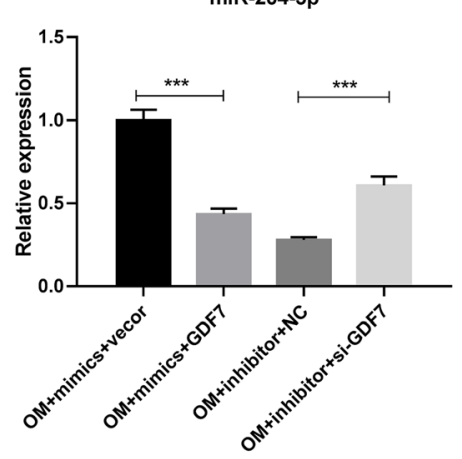

ALP

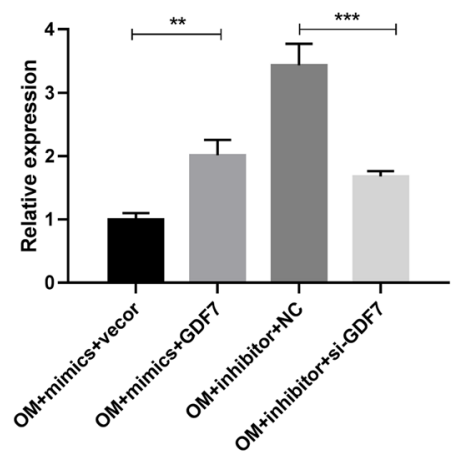

B $\mathrm{OM}+$ mimics $\mathrm{OM}+$ inhibitor

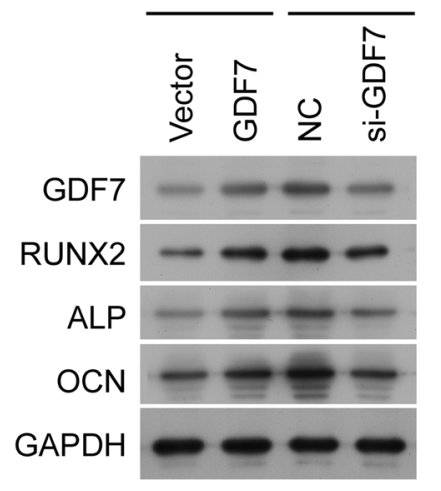

C

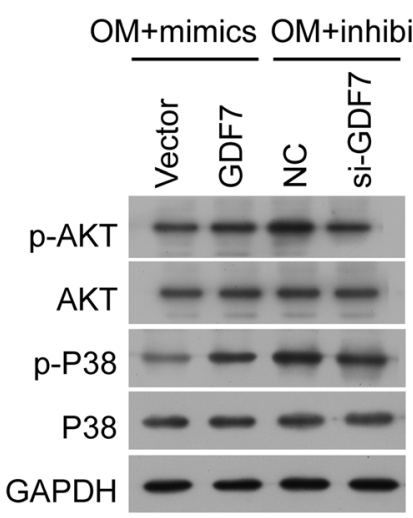

GDF7

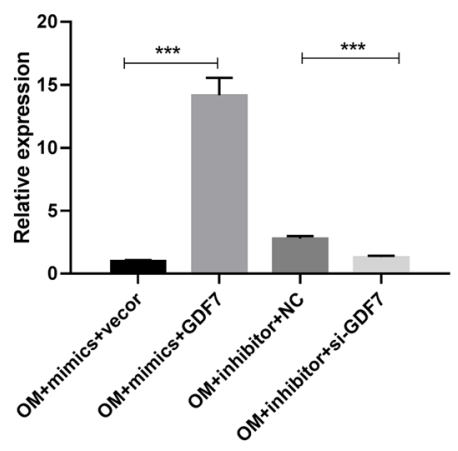

OCN

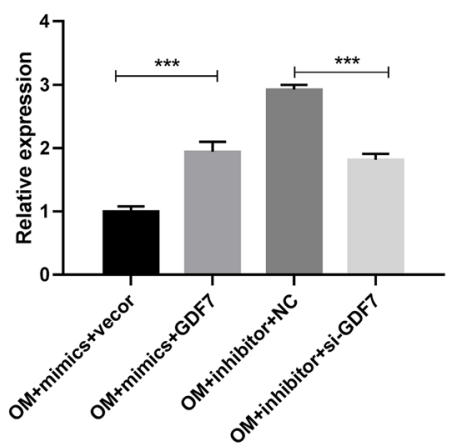

RUNX2

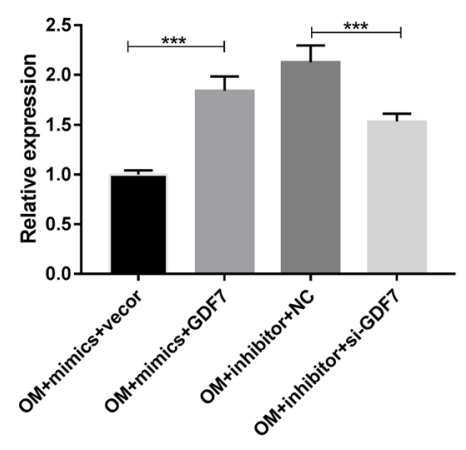

mimics+Vector mimics+GDF7 - inhibitor+NC $\square$ inhibitor+si-GDF7
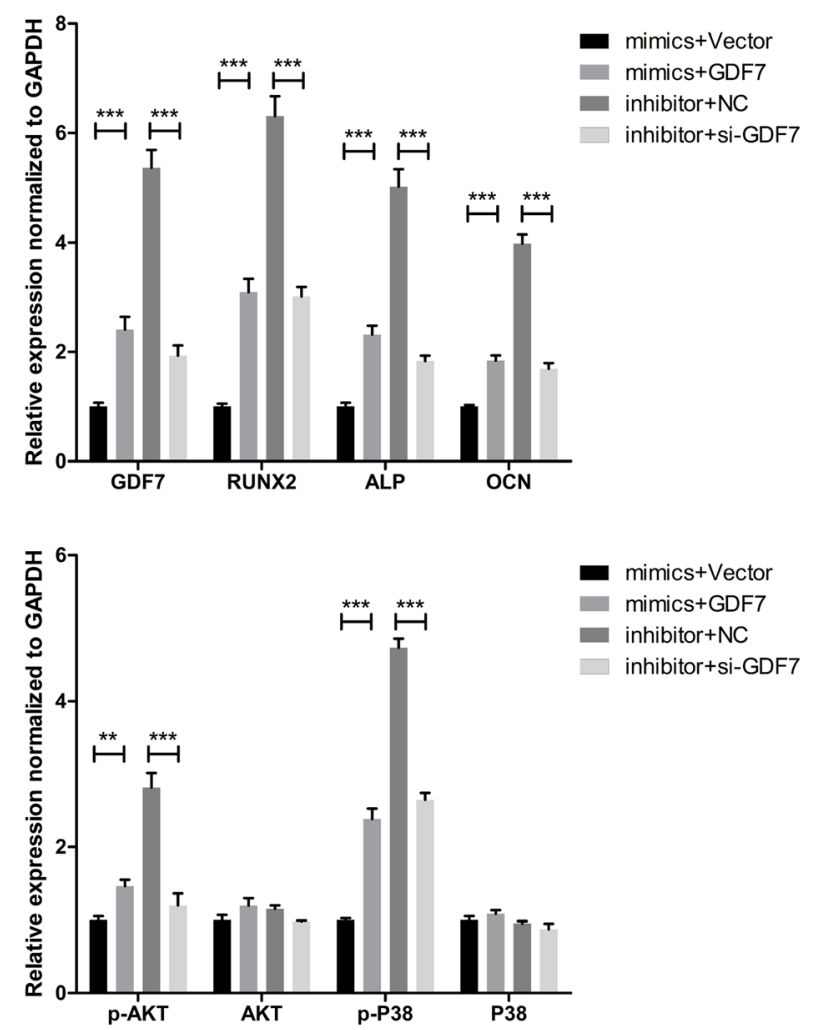

Fig. 6 (See legend on next page.) 
(See figure on previous page.)

Fig. 6 GDF7 impairs the suppressive effect of miR-204-5p on the expression of RUNX2, ALP, OCN, p-AKT and p-P38. hADSCs were co-transfected with miR-204-5p mimics and GDF7-overexpression vector or empty vector or co-transfected with miR-204-5p inhibitors and si-GDF and cultured in OM. a Relative expression of miR-204-5p, GDF7, RUNX2, ALP and OCN measured by qRT-PCR on day 14 of osteogenic induction. U6 and GAPD $\mathrm{H}$ were used as internal control genes. $\mathbf{b}$ Left panel: Western blot of GDF7, RUNX2, ALP and OCN protein levels on day 14 after osteogenic induction. GAPDH was used as an internal control. Right panel: quantitative analysis of the protein levels. c Left panel: Western blot of p-AKT, AKT, p-P38 and P38 protein levels on day 14 after osteogenic induction. GAPDH was used as an internal control. Right panel: quantitative analysis of the protein levels. Data are presented as the mean \pm SD. ${ }^{*} p<0.05,{ }^{* *} p<0.01,{ }^{* *} p<0.001$ ( $n=3$ independent experiments)

induce overexpression of osteogenic key proteins, such as RUNX2, ALP and OCN, and GDF7 inactivation led to the opposite results. Although many studies have indicated that GDF7 stimulates the proliferation of tendon fibroblasts and induces tendon and ligament formation [23, 37], Shinji et al. found that GDF7 gene transfer into a rat femoral bone defect induced tendon/ligament-like tissue formation until 4 weeks after the operation, and these tissues were eventually replaced with bone-like tissue by 8 weeks after the operation, suggesting that GDF7 initiated the replacement of tendon/ligament-like tissue with bony tissue [38]. In our study, in contrast to the main opinions, we found that GDF7 also plays an important role in regulating osteoblast differentiation and subsequent bone formation. The diverse conclusions may be due to the different species, and the lack of animal experiments might limit our insight.

Interestingly, we also found that GDF7 is a target gene of miR-204-5p and that miR-204-5p could suppress the expression of GDF7. In other words, miR-204-5p could downregulate both FOXC1 and GDF7 to inhibit the osteogenesis of hADSCs. To verify the specific regulatory mechanism among miR-204-5p, FOXC1 and GDF7 in the osteoblast differentiation of hADSCs, we performed dual-luciferase reporter assays and ChIP assays, and we found that the transcription factor FOXC1 could bind to the promoters of miR-204-5p and GDF7 and enhance the translation of GDF7, indicating that FOXC1 functioned upstream of GDF7. However, whether FOXC1 expression may be altered by GDF7, or feedback loop might exist between these proteins, should be further determined. To our surprise, our results suggested that overexpression of FOXC1 could reverse the inhibition of hADSC osteogenic differentiation by downregulating miR-204-5p, while downregulation of FOXC1 could weaken the inhibition of miR-204-5p, thus attenuating the promotion of osteogenic differentiation by the miR-204-5p inhibitor. To further investigate the regulatory mechanism between FOXC1 and miR-204-5p, we explored the production of miR-204-5p and found that FOXC1 can promote the deacetylation of the promoter region of miR-204-5p.

The transcription of miRNA is mainly performed by RNA polymerase II, and the structure of the primary transcript pri-miRNA contains the same 7-methylguanosine cap and poly(A) tail as the primary transcript of the gene encoding the protein. In addition, miRNAs are also regulated by epigenetic modifications such as DNA methylation and histone acetylation. Research has suggested that approximately $5-10 \%$ of mammalian miRNAs are regulated by epigenetics [39]. For example, the correct expression of miR-148a, miR-34b/c, miR-9 and let-7a-3 depends on the methylation status and DNMT1, DNMT3b or other DNA methylase activities [39]. Saito et al. found that when DNA demethylation reagents and histone deacetylation inhibitors were used to treat human T24 bladder cancer cells, approximately $5 \%$ of miRNAs were upregulated by more than 3 times, especially miR- 127 in CpG islands [40]. This further illustrated that the regulation of miRNA genes by epigenetic modification is in their promoter regions and can be activated by inhibiting DNA methylation and histone acetylation. However, low histone acetylation in special promoter regions often leads to gene silencing [41]. In our study, we investigated the effect of FOXC1 on the transcriptional activity of the miR-204-5p promoter by ChIP assay. The results showed that FOXC1 could weaken H3K9AC acetylation, promote HDAC2 deacetylation of the miR-204-5p promoter and reduce the transcriptional activity of miR-204-5p during osteogenic differentiation.

To date, studies have confirmed that the activation of p38 MAPK and AKT signalling pathways is the crucial stimulus for the osteogenic differentiation of hADSCs [42-44]. Our data confirmed that GDF7 overexpression can promote the phosphorylation of P38 and AKT, thus promoting the P38 and AKT signalling pathways. Moreover, this study showed that overexpression of GDF7 reversed the inhibition of osteogenic differentiation of hADSCs by miR-204-5p mimics. Additionally, FOXC1 promoted the transcriptional activity of GDF7 by inhibiting H3K9AC acetylation and promoting HDAC2 deacetylation of the miR-204-5p promoter during osteogenic differentiation.

Consistent with previous studies, we also found that miR-204-5p, FOXC1 and GDF7 play important roles during osteogenic differentiation, and we further elucidated the underlying mechanism of the regulatory network between these factors for the first time.

We summarized the mechanism of the miR-204-5p/ FOXC1/GDF7 regulatory axis during osteogenic differentiation as shown in Fig. 9. miR-204-5p is downregulated 

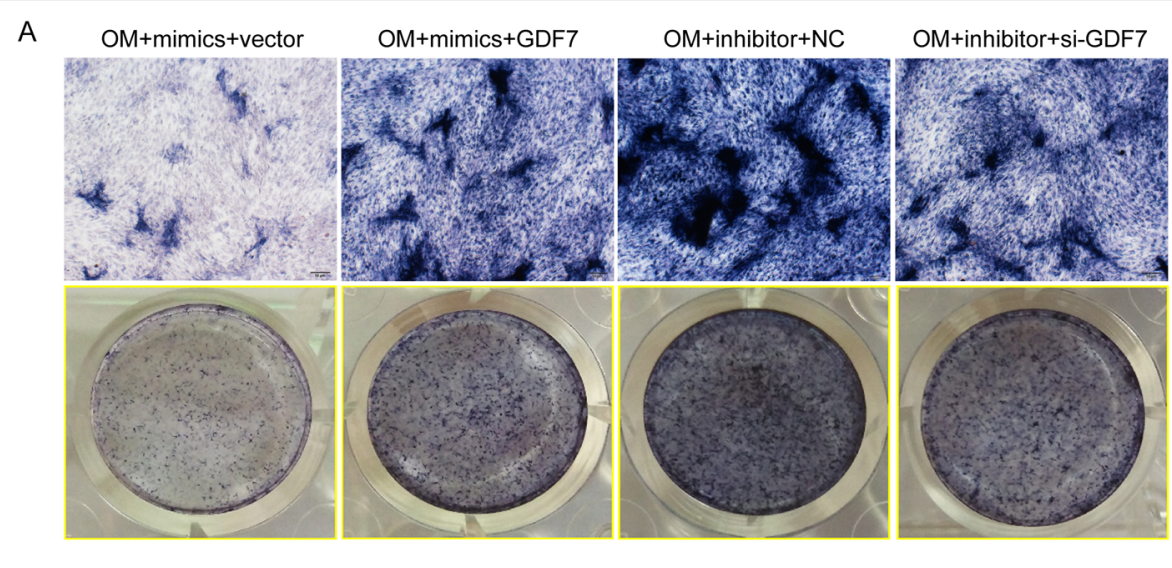

B
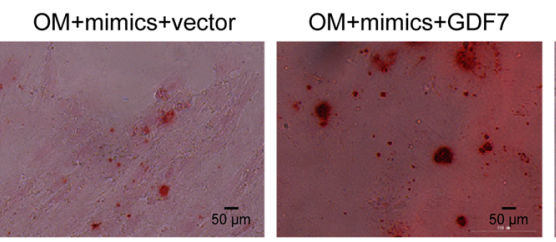

OM+inhibitor+NC
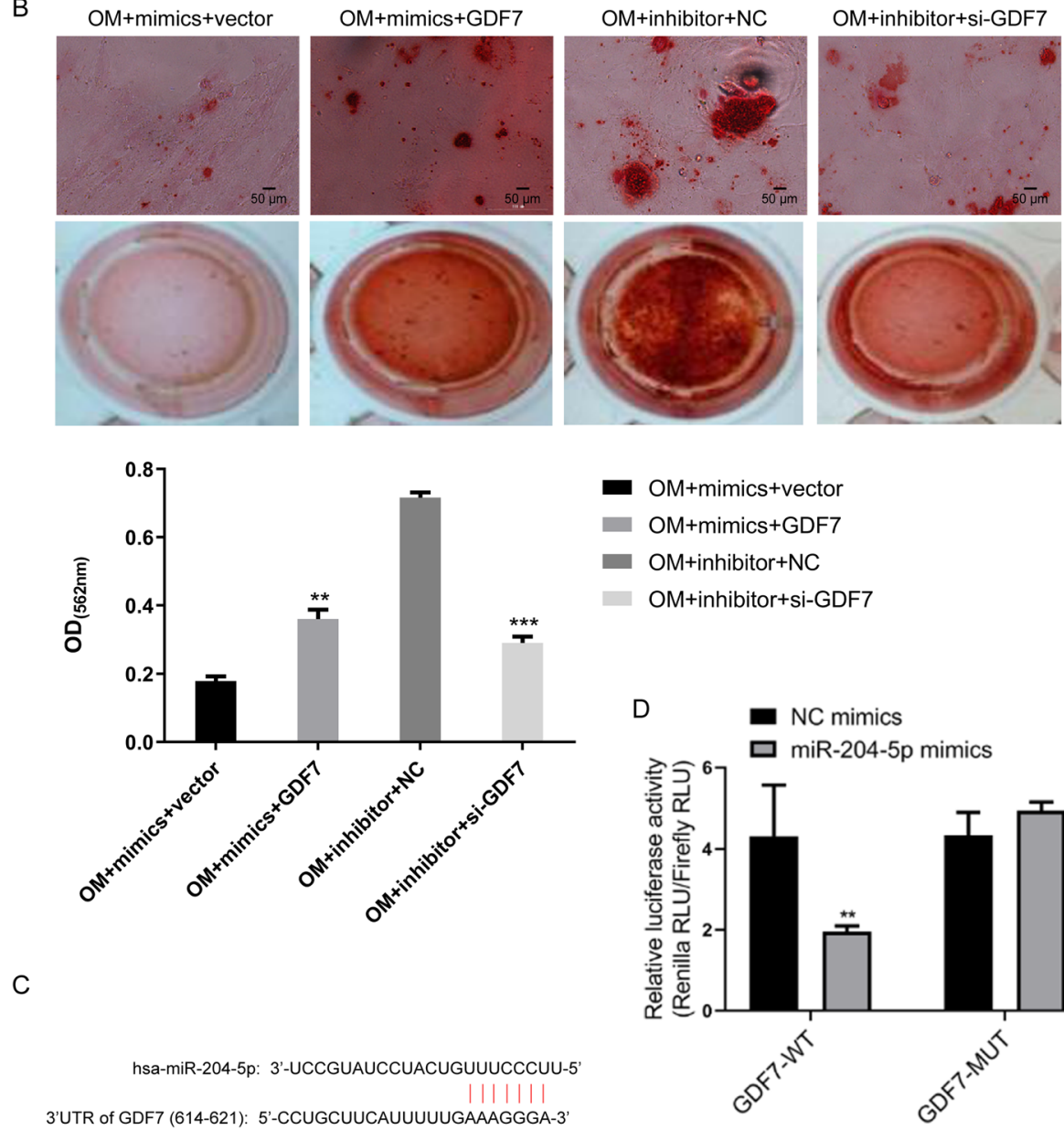

- OM+mimics+vector

- OM+mimics+GDF7

- OM+inhibitor+NC

OM+inhibitor+si-GDF7

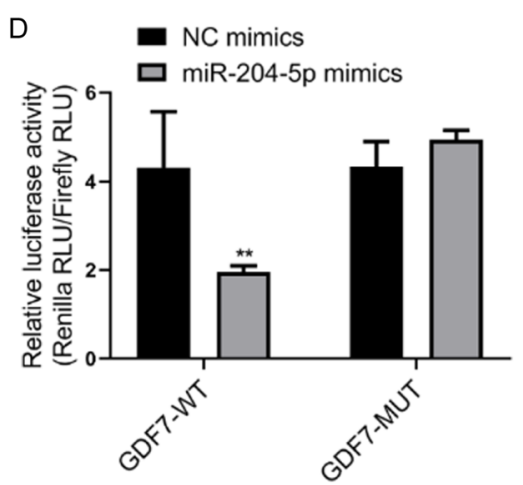

Fig. 7 GDF7 impairs the suppressive effect of miR-204-5p on the osteogenic differentiation of hADSCs. hADSCs were co-transfected with miR-204-5p mimics and GDF7-overexpression vector or empty vector or co-transfected with miR-204-5p inhibitors and si-GDF and cultured in OM. ALP staining (a) and ARS (b) on day 14 after osteogenic induction. Quantification of ARS was shown below. c Predicted binding site of miR-204-5p in the $3^{\prime}-\mathrm{UTR}$ of GDF7 mRNA. d Relative luciferase activity of cells in different groups. Data are presented as the mean \pm SD. ${ }^{*} p<0.05,{ }^{* *} p<0.01,{ }^{* * *} p<0.001(n=3$ independent experiments)

during the osteogenic differentiation of hADSCs. Moreover, miR-204-5p can bind to the 3'-UTR region of FOXC1 and GDF7 and suppress the expression of FOXC1 and GDF7. FOXC1 bound to the promoters of miR-204- 5p and GDF7, promoted the deacetylation of miR-204-5p and reduced the expression of miR-204-5p, thus promoting the expression of GDF7, which induced hADSC osteogenesis by activating the AKT and P38 signalling 


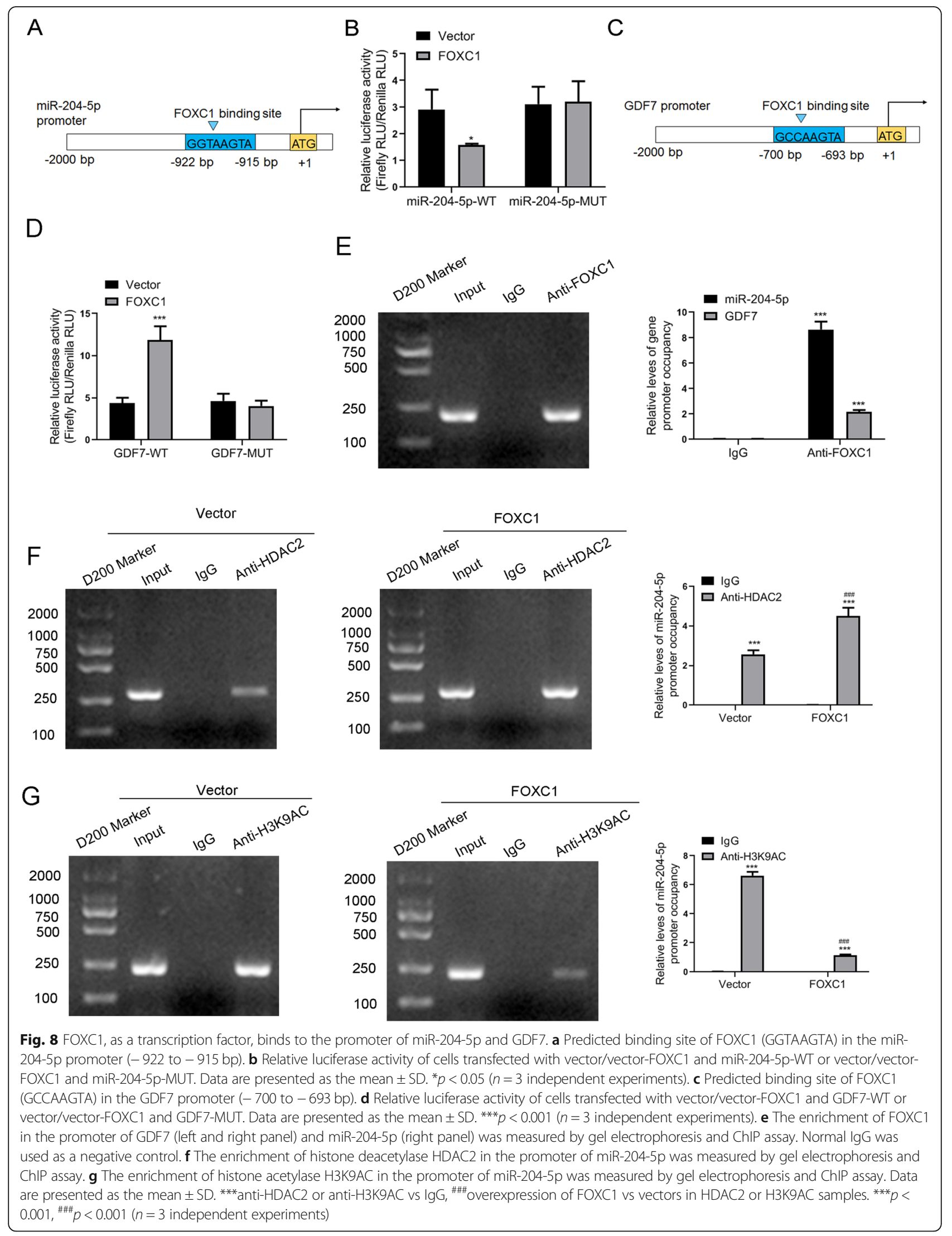




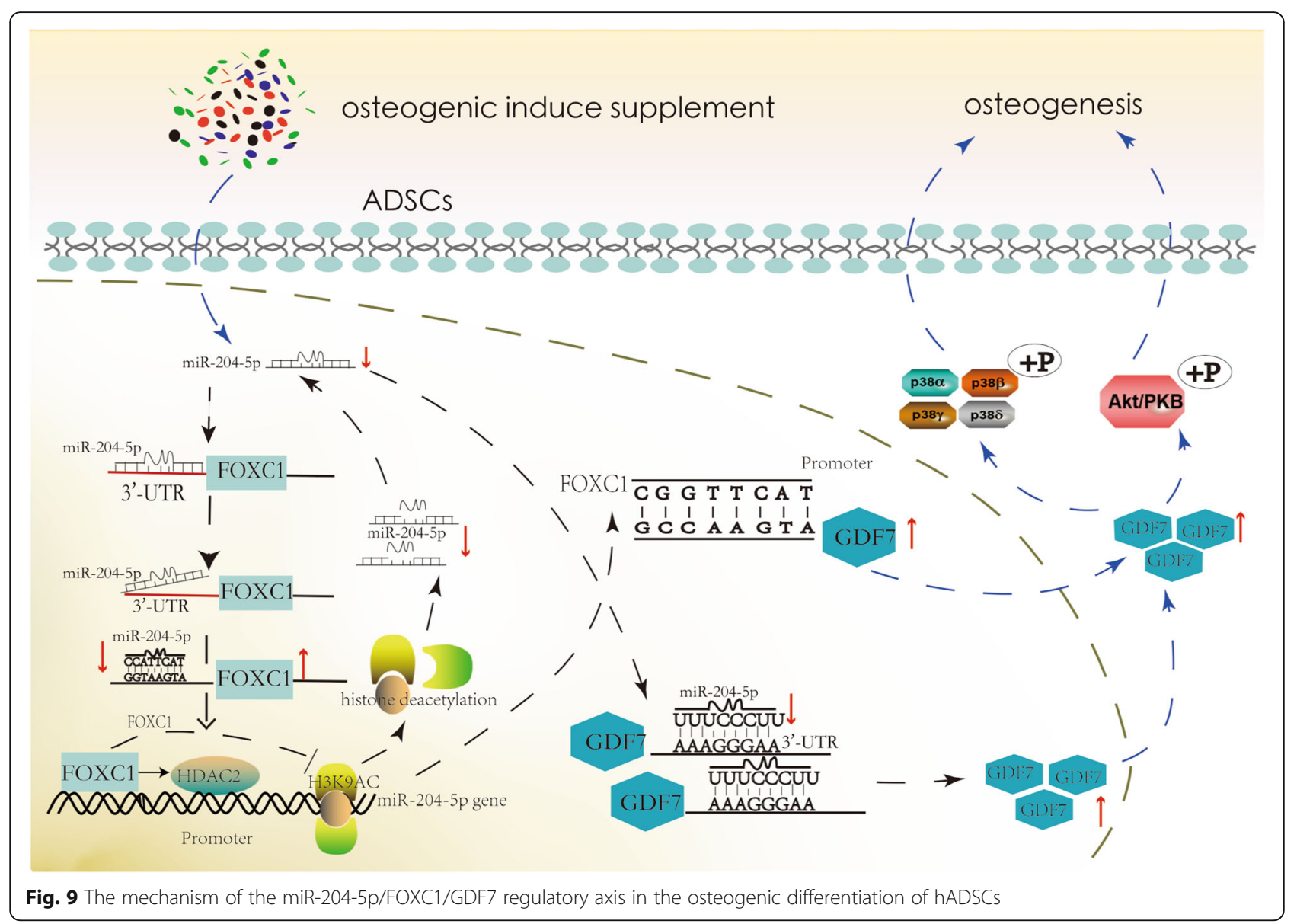

pathways. Finally, the FOXC1/GDF7/miR-204-5p axis formed a feedback loop, which plays an important role in the balance of osteogenic differentiation. The specific mechanisms of downstream signalling pathways of GDF7 and whether FOXC1 can affect the methylation of the miR-204-5p promoter will be our future research focuses.

Modification of the miRNAs that mediate osteogenic differentiation has become an approach to optimize the osteogenic potential of hADSCs. One strategy is to either expose the cells to inhibitors of these miRNAs or to engineer cells that lack expression of these miRNAs. Another is to target the upstream factors that regulate these miRNAs. Since we found that miR-204-5p was downregulated during the osteogenic differentiation of hADSCs and that FOXC1 could bind to the promoter of miR204-5p, functioning as an upstream mediator of miR204-5p, modification of the expression of miR-204-5p of FOXC1 could be an efficient approach in bone tissue engineering and bone healing therapies.

\section{Conclusion}

Our results demonstrated that the miR-204-5p/FOXC1/ GDF7 axis regulates the osteogenic differentiation of
hADSCs via the AKT and p38 signalling pathways. This study further revealed the regulatory mechanism of hADSC differentiation from the perspective of miRNA regulation.

\section{Abbreviations}

hADSCs: Human adipose-derived stem cells; FOXC1: Forkhead box C1; GDF7: Growth differentiation factor 7; BMP12: Bone morphogenetic protein 12; CDMP3: Cartilage-derived morphogenetic protein 3; miRNA: MicroRNA; MSCs: Mesenchymal stem cells; TGF- $\beta$ : Transforming growth factor beta; PBS: Phosphate-buffered saline; DMEM F12: Dulbecco's modified Eagle's medium/Nutrient F-12; FBS: Foetal bovine serum; PM: Proliferation medium; OM: Osteogenic differentiation medium; ALP: Alkaline phosphatase; ARS: Alizarin red staining; DAPI: 40,6-Diamidino-2-phenylindole; WT: Wildtype; MUT: Mutant-type; ChIP: Chromatin immunoprecipitation; IgG: Immunoglobulin G; 3'-UTR: 3' untranslated region; CXCL: CXC chemokine ligand; CAR cells: CXCL 12-abundant reticular cells

\section{Acknowledgements}

The authors acknowledge the patients who donated the adipose tissue at the Department of Plastic Surgery, The First Hospital of China Medical University. This work was sponsored by the National Natural Science Foundation of China (51872332).

\section{Authors' contributions}

You Zhou and Shu Guo contributed to the research conception and design. You Zhou, Siyu Liu and Wei Wang performed the experiments and analysed and interpreted the data. Qiang Sun and Mengzhu Lv obtained adipose tissue and helped with the cell culture. Shude Yang and Shuang Tong 
interpreted the data and helped with the statistical analysis. You Zhou drafted the manuscript. Shu Guo revised the manuscript critically. All authors approved the final manuscript

\section{Funding}

This work was sponsored by the National Natural Science Foundation of China (51872332).

\section{Availability of data and materials}

Please contact the authors for data requests.

\section{Ethics approval and consent to participate}

The above experimental samples were collected with the informed consen of the clinical patients and approved by the Ethics Committee of the First Hospital of China Medical University (AF-SOP-07-1.1-01)

\section{Consent for publication}

Not applicable.

\section{Competing interests}

The authors confirm that there are no conflicts of interest.

\section{Author details}

${ }^{1}$ Department of Plastic Surgery, The First Hospital of China Medical University, NO 155 Nanjing street Heping Strict, Shenyang 110001, Liaoning Province, China. ${ }^{2}$ Institute of Respiratory Disease, The First Hospital of China Medical University, NO 155 Nanjing street Heping Strict, Shenyang 110001, Liaoning Province, China.

\section{Received: 3 September 2020 Accepted: 22 December 2020}

\section{Published online: 18 January 2021}

\section{References}

1. Vacanti JP, Langer R. Tissue engineering: the design and fabrication of living replacement devices for surgical reconstruction and transplantation. Lancet. 1999:354(Suppl 1):SI32-4

2. Jin Q, Yuan K, Lin W, Niu C, Ma R, Huang Z. Comparative characterization of mesenchymal stem cells from human dental pulp and adipose tissue for bone regeneration potential. Artif Cells Nanomed Biotechnol. 2019;47(1): 1577-84.

3. Zuk PA, Zhu M, Ashjian P, et al. Human adipose tissue is a source of multipotent stem cells. Mol Biol Cell. 2002;13(12):4279-95.

4. Stan Gronthos, Dawn M. Franklin, Holly A. Leddy, Pamela G. Robey, Robert W. Storms, Jeffrey M. Gimble. Surface protein characterization of human adipose tissue-derived stromal cells. J Cell Physiol 2001;189(1):54-63.

5. De Francesco F, Ricci G, D'Andrea F, Nicoletti GF, Ferraro GA. Human adipose stem cells: from bench to bedside. Tissue Eng B Rev. 2015;21(6): 572-84

6. Mohr AM, Mott JL. Overview of microRNA biology. Semin Liver Dis. 2015; 35(1):3-11.

7. Catalanotto C, Cogoni C, Zardo G. MicroRNA in control of gene expression: an overview of nuclear functions. Int J Mol Sci. 2016;17(10):1712

8. Dong $H$, Lei J, Ding L, Wen $Y$, Ju H, Zhang X. MicroRNA: function, detection, and bioanalysis. Chem Rev. 2013;113(8):6207-33.

9. Lu TX, Rothenberg ME. MicroRNA. J Allergy Clin Immunol. 2018;141(4):1202-7.

10. Li B. MicroRNA regulation in osteogenic and adipogenic differentiation of bone mesenchymal stem cells and its application in bone regeneration. Curr Stem Cell Res Ther. 2018;13(1):26-30.

11. Hodges WM, O'Brien F, Fulzele S, Hamrick MW. Function of microRNAs in the osteogenic differentiation and therapeutic application of adiposederived stem cells (ASCS). Int J Mol Sci. 2017;18(12):2597.

12. Yang $Y$, Huang G, Zhou Z, Fewell JG, Kleinerman ES. miR-20a regulates FAS expression in osteosarcoma cells by modulating FAS promoter activity and can be therapeutically targeted to inhibit lung metastases. Mol Cancer Ther. 2018;17(1):130-9.

13. Godfrey TC, Wildman BJ, Beloti MM, et al. The microRNA-23a cluster regulates the developmental HoxA cluster function during osteoblast differentiation. J Biol Chem. 2018:293(45):17646-60.

14. Grieco FA, Schiavo AA, Brozzi F, et al. The microRNAs miR-211-5p and miR204-5p modulate ER stress in human beta cells. J Mol Endocrinol. 2019. 63(2):139-149
15. Yu C, Li L, Xie F, et al. LncRNA TUG1 sponges miR-204-5p to promote osteoblast differentiation through upregulating Runx2 in aortic valve calcification. Cardiovasc Res. 2018;114(1):168-79.

16. Elian FA, Yan E, Walter MA. FOXC1, the new player in the cancer sandbox. Oncotarget. 2018;9(8):8165-78.

17. Han B, Bhowmick N, Qu Y, Chung S, Giuliano AE, Cui X. FOXC1: an emerging marker and therapeutic target for cancer. Oncogene. 2017;36(28): 3957-63.

18. Jingjing Sun, Mamoru Ishii, Man-Chun Ting, Robert Maxson. Foxc1 controls the growth of the murine frontal bone rudiment by direct regulation of a Bmp response threshold of Msx2. Dev. 2013;140(5):1034-1044.

19. Kume T, Deng KY, Winfrey V, Gould DB, Walter MA, Hogan BL. The forkhead/winged helix gene Mf1 is disrupted in the pleiotropic mouse mutation congenital hydrocephalus. Cell. 1998;93(6):985-96.

20. Rice R, Rice DP, Olsen BR, Thesleff I. Progression of calvarial bone development requires Foxc1 regulation of Msx2 and Alx4. Dev Biol. 2003; 262(1):75-87.

21. Michiko Yoshida, Kenji Hata, Rikako Takashima, et al. The transcription factor Foxc1 is necessary for Ihh-Gli2-regulated endochondral ossification. Nat Commun. 2015;6:6653

22. Chang SC, Hoang B, Thomas JT, et al. Cartilage-derived morphogenetic proteins. New members of the transforming growth factor-beta superfamily predominantly expressed in long bones during human embryonic development. J Biol Chem. 1994;269(45):28227-34.

23. Wolfman NM, Hattersley G, Cox K, et al. Ectopic induction of tendon and ligament in rats by growth and differentiation factors 5,6 , and 7 , members of the TGF-beta gene family. J Clin Invest. 1997;100(2):321-30.

24. Shen H, Gelberman RH, Silva MJ, Sakiyama-Elbert SE, Thomopoulos S. BMP12 induces tenogenic differentiation of adipose-derived stromal cells. Plos One. 2013;8(10):e77613.

25. He $\mathrm{H}$, Chen $\mathrm{K}$, Wang $\mathrm{F}$, et al. miR-204-5p promotes the adipogenic differentiation of human adipose-derived mesenchymal stem cells by modulating DVL3 expression and suppressing Wnt/ß-catenin signaling. Int J Mol Med. 2015:35(6):1587-95.

26. Hopkins A, Mirzayans F, Berry F. Foxc1 expression in early osteogenic differentiation is regulated by BMP4-SMAD activity. J Cell Biochem. 2016: 117(7):1707-17.

27. Maria AT, Maumus M, Le Quellec A, Jorgensen C, Noel D, Guilpain P. Adipose-derived mesenchymal stem cells in autoimmune disorders: state of the art and perspectives for systemic sclerosis. Clin Rev Allergy Immunol. 2017:52(2):234-59.

28. Mahmoudifar N, Doran PM. Mesenchymal stem cells derived from human adipose tissue. Methods Mol Biol (Clifton). 2015:1340:53-64.

29. Huang S, Wang S, Bian C, et al. Upregulation of miR-22 promotes osteogenic differentiation and inhibits adipogenic differentiation of human adipose tissue-derived mesenchymal stem cells by repressing HDAC6 protein expression. Stem Cells Dev. 2012;21(13):2531-40.

30. Shen H, Lu C, Shi J, Li H, Si J, Shen G. Satb2 expression in Foxc1-promoted osteogenic differentiation of MC3T3-E1 cells is negatively regulated by microRNA-103-3p. Acta Biochim Biophys Sin. 2019;51(6):588-97.

31. Gong $Y, X u F$, Zhang $L$, et al. MicroRNA expression signature for Satb2induced osteogenic differentiation in bone marrow stromal cells. Mol Cell Biochem. 2014:387(1-2):227-39.

32. Ouyang $\mathrm{N}$, Li H, Wang M, Shen H, Si J, Shen G. The transcription factor Foxc1 promotes osteogenesis by directly regulating Runx2 in response of intermittent parathyroid hormone (1-34) treatment. Front Pharmacol. 2020; 11:592.

33. Wang $L$, Siegenthaler JA, Dowell RD, Yi R. Foxc1 reinforces quiescence in self-renewing hair follicle stem cells. Science. 2016;351(6273):613-7.

34. Caddy JC, Luoma LM, Berry FB. FOXC1 negatively regulates BMP-SMAD activity and Id 1 expression during osteoblast differentiation. J Cell Biochem. 2020;121(5-6):3266-77.

35. Yang L, Liang $H$, Meng $X$, et al. mmu_circ_0000790 is involved in pulmonary vascular remodeling in mice with $\mathrm{HPH}$ via microRNA-374cmediated FOXC1. Mol Ther Nucleic Acids. 2020;20:292-307.

36. Wang DS, Zhang HQ, Zhang B, et al. miR-133 inhibits pituitary tumor cell migration and invasion via down-regulating FOXC1 expression. Genet Mol Res. 2016:15(1): gmr.15017453.

37. Fu SC, Wong YP, Chan BP, et al. The roles of bone morphogenetic protein (BMP) 12 in stimulating the proliferation and matrix production of human patellar tendon fibroblasts. Life Sci. 2003;72(26):2965-74. 
38. Kuroda S, Goto N, Suzuki M, et al. Regeneration of bone- and tendon/ ligament-like tissues induced by gene transfer of bone morphogenetic protein-12 in a rat bone defect. J Tissue Eng. 2010;2010:891049.

39. Brueckner B, Stresemann C, Kuner R, et al. The human let-7a-3 locus contains an epigenetically regulated microRNA gene with oncogenic function. Cancer Res. 2007;67(4):1419-23.

40. Saito $Y$, Liang G, Egger $G$, et al. Specific activation of microRNA-127 with downregulation of the proto-oncogene BCL6 by chromatin-modifying drugs in human cancer cells. Cancer Cell. 2006;9(6):435-43.

41. Yin $L H$, Zheng $X Q, L i H Y$, et al. Epigenetic deregulated miR-375 contributes to the constitutive activation of JAK2/STAT signaling in myeloproliferative neoplasm. Leuk Res. 2015;39(4):471-8.

42. Jiang Y, Wu W, Jiao G, Chen Y, Liu H. LncRNA SNHG1 modulates p38 MAPK pathway through Nedd4 and thus inhibits osteogenic differentiation of bone marrow mesenchymal stem cells. Life Sci. 2019;228:208-14.

43. Ba P, Duan X, Fu G, Lv S, Yang P, Sun Q. Differential effects of p38 and Erk1/ 2 on the chondrogenic and osteogenic differentiation of dental pulp stem cells. Mol Med Rep. 2017;16(1):63-8.

44. Ohashi E, Kohno K, Arai N, Harashima A, Ariyasu T, Ushio S. Adenosine N1oxide exerts anti-inflammatory effects through the PI3K/Akt/GSK-3 $\beta$ signaling pathway and promotes osteogenic and adipocyte differentiation. Biol Pharm Bull. 2019:42(6):968-76.

\section{Publisher's Note}

Springer Nature remains neutral with regard to jurisdictional claims in published maps and institutional affiliations.

Ready to submit your research? Choose BMC and benefit from:

- fast, convenient online submission

- thorough peer review by experienced researchers in your field

- rapid publication on acceptance

- support for research data, including large and complex data types

- gold Open Access which fosters wider collaboration and increased citations

- maximum visibility for your research: over $100 \mathrm{M}$ website views per year

At $\mathrm{BMC}$, research is always in progress.

Learn more biomedcentral.com/submissions 